The Structural and Conformational Properties of 2-Propenylgermane

(Allylgermane) Studied by Microwave Spectroscopy and Quantum Chemical

\title{
Calculations
}

(Supporting Information)

\author{
Anne Horn, ${ }^{\dagger}$ Harald Møllendal* ${ }^{\dagger}{ }^{\dagger}$ Jean Demaison, ${ }^{\ddagger}$ Denis Petitprez,${ }^{\ddagger}$ Juan Ramon Aviles \\ Moreno, ${ }^{\dagger}$ Abdessamad Benidar ${ }^{\S}$ and Jean-Claude Guillemin ${ }^{\#}$
}

Department of Chemistry, University of Oslo, P. O. Box 1033 Blindern, NO-0315 Oslo, Norway, Laboratoire de Physique des Lasers, Atomes et Molécules, UMR CNRS 8523, Bât. P5, Université de Lille1, F-59655 Villeneuve d'Ascq, France, Equipe d'Astrochimie Expérimentale, Laboratoire PALMS, UMR CNRS 6627, Université de Rennes I, Campus de Beaulieu, F-35042 France, Laboratoire de Synthèse et Activation de Biomolécules, UMR CNRS 6052, Institut de Chimie de Rennes, ENSCR, F-35700 Rennes, France

*Corresponding author. E-mail: harald.mollendal@kjemi.uio.no. Tel: +47 228556 74, Fax: +47 22 855441.

${ }^{\dagger}$ University of Oslo

†Université de Lille 1

${ }^{\S}$ Université de Rennes 1

${ }^{\#}$ ENSCR 
TABLE 1S: Allylgermane-74. Ground vibrational state.

Calculation by ROTFIT-TPW-1.5 of 29-MAY-1995 (with weights)

Watson A-reduction:

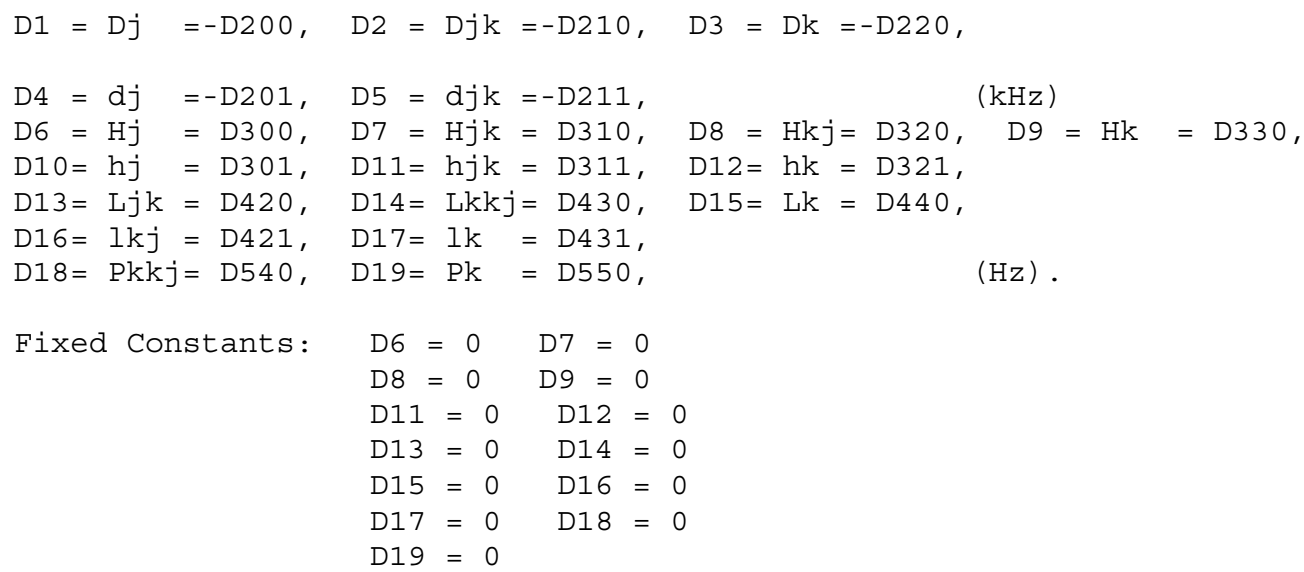

Total number of transitions: 167

Transition obs. frequency obs.- weight $t$ distortion corrections calc.

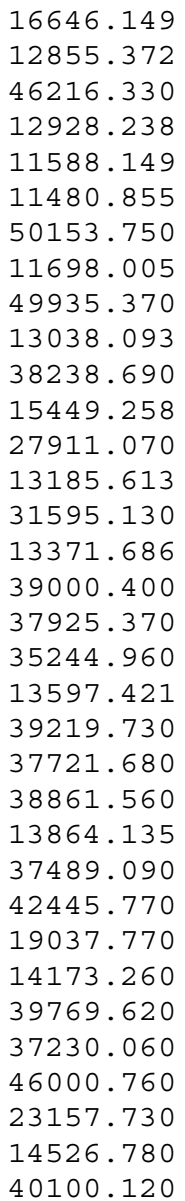

$\begin{array}{rrr}-0.010 & 0.0030 & 9.9\end{array}$ $-0.0130 .0030-8.3$

$\begin{array}{llll}0.037 & 0.050 & 0.3\end{array}$

$\begin{array}{llll}-0.010 & 0.0030 & -3.9\end{array}$

$\begin{array}{llll}0.009 & 0.0030 & 9.9\end{array}$

$\begin{array}{lll}0.011 & 0.0030 & 9.9\end{array}$

$\begin{array}{llll}-0.037 & 0.050 & -0.3\end{array}$

$\begin{array}{llll}0.010 & 0.0030 & 9.9\end{array}$

$\begin{array}{llll}0.301 & 0.050 & 2.2\end{array}$

$-0.0100 .0030-3.1$

$\begin{array}{llll}0.083 & 0.050 & 0.6\end{array}$

$\begin{array}{lll}0.012 & 0.0030 & 9.9\end{array}$

$\begin{array}{llll}0.019 & 0.050 & 0.1\end{array}$

$\begin{array}{llll}-0.008 & 0.0030 & -2.2\end{array}$

$\begin{array}{lll}0.032 & 0.050 & 0.2\end{array}$

$\begin{array}{llll}-0.006 & 0.0030 & -2.1\end{array}$

$\begin{array}{llll}0.133 & 0.050 & 1.0\end{array}$

$\begin{array}{lll}-0.046 & 0.050 & -0.3\end{array}$

$0.076 \quad 0.050 \quad 0.6$

$\begin{array}{lll}-0.003 & 0.0030 & 9.9\end{array}$

$\begin{array}{lll}-0.059 & 0.050 & -0.4\end{array}$

$\begin{array}{lll}-0.007 & 0.050 & -0.1\end{array}$

$\begin{array}{lll}0.072 & 0.050 & 0.5\end{array}$

$\begin{array}{lll}-0.000 & 0.0030 & 9.9\end{array}$

$\begin{array}{llll}-0.031 & 0.050 & -0.2\end{array}$

$\begin{array}{lll}-0.450 & 0.050 & -3.3\end{array}$

$\begin{array}{llll}-0.242 & 0.050 & -1.8\end{array}$

$\begin{array}{llll}-0.087 & 0.050 & -0.6\end{array}$

$0.0710 .050 \quad 0.5$

$\begin{array}{lll}-0.011 & 0.050 & -0.1\end{array}$

$\begin{array}{lll}0.131 & 0.050 & 1.0\end{array}$

$\begin{array}{llll}-0.011 & 0.050 & -0.1\end{array}$

$0.006 \quad 0.050 \quad 0.0$

$\begin{array}{lll}0.092 & 0.050 & 0.7\end{array}$

\begin{abstract}
total higher fixed
\end{abstract}

$\begin{array}{rr}-0.314 & -2.1 \mathrm{E}-8 \\ -0.317 & 2.1 \mathrm{E}-8 \\ -5.071 & 2.4 \mathrm{E}-8 \\ -0.168 & 5.7 \mathrm{E}-7 \\ -0.171 & -6.1 \mathrm{E}-8 \\ 0.109 & -4.0 \mathrm{E}-6 \\ -4.390 & 6.3 \mathrm{E}-7 \\ 0.038 & 4.0 \mathrm{E}-6 \\ -4.351 & -5.7 \mathrm{E}-7 \\ 0.041 & 4.6 \mathrm{E}-6 \\ -4.390 & -4.5 \mathrm{E}-6 \\ -0.403 & -4.7 \mathrm{E}-7 \\ 0.143 & -2.1 \mathrm{E}-5 \\ 0.292 & 2.1 \mathrm{E}-5 \\ 0.296 & -7.1 \mathrm{E}-5 \\ 0.562 & 7.3 \mathrm{E}-5 \\ -2.500 & 7.1 \mathrm{E}-5 \\ -1.993 & -6.8 \mathrm{E}-5 \\ 0.425 & -1.9 \mathrm{E}-4 \\ 0.819 & 2.0 \mathrm{E}-4 \\ -1.251 & 2.0 \mathrm{E}-4 \\ -0.325 & -1.8 \mathrm{E}-4 \\ 0.521 & -4.6 \mathrm{E}-4 \\ 1.022 & 4.9 \mathrm{E}-4 \\ 1.685 & -4.2 \mathrm{E}-4 \\ 0.578 & -9.7 \mathrm{E}-4 \\ -5.830 & 3.7 \mathrm{E}-4 \\ 1.121 & 1.1 \mathrm{E}-3 \\ 1.578 & 9.9 \mathrm{E}-4 \\ 4.058 & -8.8 \mathrm{E}-4 \\ 0.594 & -1.9 \mathrm{E}-3 \\ -8.242 & 7.7 \mathrm{E}-4 \\ 1.057 & 2.1 \mathrm{E}-3 \\ 3.073 & 1.9 \mathrm{E}-3 \\ & \end{array}$




\begin{tabular}{|c|c|c|c|c|c|c|c|c|c|c|c|}
\hline 9 & 1 & 8 & 9 & 2 & 7 & 36947.250 & 0.031 & 0.050 & 0.2 & 6.815 & $-1 \cdot 7 E-3$ \\
\hline 9 & 0 & 9 & 10 & 1 & 10 & 49526.560 & 0.069 & 0.050 & 0.5 & 0.563 & $-3.4 E-3$ \\
\hline 9 & 1 & 9 & 10 & 0 & 10 & 7302.080 & 0.007 & 0.050 & 0.1 & -11.204 & $1.4 E-3$ \\
\hline 10 & 0 & 10 & 10 & 1 & 9 & 4926.230 & -0.078 & 0.050 & -0.6 & 0.757 & $3.9 E-3$ \\
\hline 10 & 1 & 10 & 10 & 2 & 9 & 40467.700 & -0.047 & 0.050 & -0.3 & 4.556 & $3.5 E-3$ \\
\hline 10 & 1 & 9 & 10 & 2 & 8 & 36643.620 & 0.058 & 0.050 & 0.4 & 9.968 & $-2.9 E-3$ \\
\hline 10 & 1 & 10 & 11 & 0 & 11 & 31468.610 & -0.014 & 0.050 & -0.1 & -14.768 & $2.5 E-3$ \\
\hline 11 & 0 & 11 & 11 & 1 & 10 & 5373.960 & -0.045 & 0.050 & -0.3 & 0.136 & $6.9 E-3$ \\
\hline 11 & 1 & 10 & 11 & 2 & 9 & 36322.440 & 0.037 & 0.050 & 0.3 & 13.525 & $-4.8 E-3$ \\
\hline 11 & 1 & 11 & 12 & 0 & 12 & 35654.840 & 0.016 & 0.050 & 0.1 & -18.982 & $4.2 \mathrm{E}-3$ \\
\hline 12 & 3 & 10 & 11 & 4 & 7 & 43326.450 & 0.032 & 0.050 & 0.3 & -29.025 & $-1.9 E-4$ \\
\hline 12 & 3 & 9 & 11 & 4 & 8 & 43322.750 & 0.374 & 0.050 & 3.6 & -28.995 & $-2 \cdot 3 E-4$ \\
\hline 12 & 0 & 12 & 12 & 1 & 11 & 15871.990 & -0.064 & 0.050 & -0.5 & -0.908 & 0.012 \\
\hline 12 & 1 & 12 & 12 & 2 & 11 & 41315.470 & 0.037 & 0.050 & 0.3 & 7.249 & 0.010 \\
\hline 12 & 1 & 12 & 12 & 2 & 11 & 41315.470 & 0.037 & 0.050 & 0.3 & 7.249 & 0.010 \\
\hline 12 & 1 & 11 & 12 & 2 & 10 & 35987.400 & 0.062 & 0.050 & 0.5 & 17.485 & $-7.6 E-3$ \\
\hline 12 & 1 & 12 & 13 & 0 & 13 & 39857.910 & -0.024 & 0.050 & -0.2 & -23.885 & $6.5 E-3$ \\
\hline 13 & 0 & 13 & 13 & 1 & 12 & 16422.740 & -0.015 & 0.050 & -0.1 & -2.489 & 0.019 \\
\hline 13 & 1 & 13 & 13 & 2 & 12 & 41795.840 & 0.182 & 0.050 & 1.3 & 8.325 & 0.016 \\
\hline 13 & 1 & 12 & 13 & 2 & 11 & 35642.340 & 0.113 & 0.050 & 0.8 & 21.831 & -0.011 \\
\hline 13 & 1 & 13 & 14 & 0 & 14 & 44075.120 & 0.045 & 0.050 & 0.3 & -29.509 & $9.8 E-3$ \\
\hline 14 & 3 & 12 & 13 & 4 & 9 & 35604.030 & -0.080 & 0.050 & -0.8 & -9.639 & $-5.6 E-4$ \\
\hline 14 & 3 & 11 & 13 & 4 & 10 & 35594.110 & -0.022 & 0.050 & -0.2 & -9.542 & $-7.5 E-4$ \\
\hline 14 & 0 & 14 & 14 & 1 & 13 & 17028.450 & -0.033 & 0.050 & -0.2 & -4.740 & 0.030 \\
\hline 14 & 1 & 14 & 14 & 2 & 13 & 42313.570 & -0.057 & 0.050 & -0.4 & 9.123 & 0.025 \\
\hline 14 & 1 & 13 & 14 & 2 & 12 & 35291.360 & 0.183 & 0.050 & 1.4 & 26.537 & -0.017 \\
\hline 14 & 1 & 14 & 15 & 0 & 15 & 48303.180 & -0.079 & 0.050 & -0.6 & -35.878 & 0.014 \\
\hline 15 & 0 & 15 & 15 & 1 & 14 & 17691.630 & -0.017 & 0.050 & -0.1 & -7.809 & 0.045 \\
\hline 15 & 1 & 14 & 15 & 2 & 13 & 34938.560 & 0.057 & 0.050 & 0.4 & 31.556 & -0.023 \\
\hline 15 & 2 & 14 & 16 & 1 & 15 & 28084.520 & -0.104 & 0.050 & -0.8 & -64.426 & 0.050 \\
\hline 16 & 3 & 14 & 15 & 4 & 11 & 27880.640 & 0.066 & 0.050 & 0.6 & 14.257 & $-1.4 E-3$ \\
\hline 16 & 3 & 13 & 15 & 4 & 12 & 27858.770 & 0.026 & 0.050 & 0.2 & 14.531 & $-2.1 E-3$ \\
\hline 16 & 0 & 16 & 16 & 1 & 15 & 18414.580 & -0.065 & 0.050 & -0.5 & -11.863 & 0.067 \\
\hline 16 & 1 & 15 & 16 & 2 & 14 & 34588.810 & 0.113 & 0.050 & 0.8 & 36.823 & -0.031 \\
\hline 17 & 0 & 17 & 17 & 1 & 16 & 19199.810 & -0.003 & 0.050 & -0.0 & -17.084 & 0.098 \\
\hline 17 & 1 & 17 & 17 & 2 & 16 & 44095.050 & 0.118 & 0.050 & 0.9 & 8.997 & 0.078 \\
\hline 17 & 1 & 16 & 17 & 2 & 15 & 34246.520 & 0.136 & 0.050 & 1.0 & 42.253 & -0.041 \\
\hline 17 & 2 & 16 & 18 & 1 & 17 & 36977.550 & -0.209 & 0.050 & -1.7 & -92.181 & 0.097 \\
\hline 18 & 0 & 18 & 18 & 1 & 17 & 20049.340 & -0.029 & 0.050 & -0.2 & -23.668 & 0.140 \\
\hline 18 & 1 & 18 & 18 & 2 & 17 & 44764.790 & 0.092 & 0.050 & 0.7 & 7.805 & 0.109 \\
\hline 18 & 1 & 17 & 18 & 2 & 16 & 33916.450 & 0.173 & 0.050 & 1.3 & 47.739 & -0.052 \\
\hline 18 & 2 & 17 & 19 & 1 & 18 & 41465.460 & -0.149 & 0.050 & -1.2 & -108.508 & 0.132 \\
\hline 18 & 2 & 16 & 19 & 1 & 19 & 26399.130 & -0.083 & 0.050 & -0.7 & -28.240 & -0.201 \\
\hline 19 & 4 & 15 & 18 & 5 & 14 & 41912.050 & -0.061 & 0.050 & 9.9 & -0.412 & $-3.2 E-3$ \\
\hline 19 & 0 & 19 & 19 & 1 & 18 & 20965.350 & -0.010 & 0.050 & -0.1 & -31.827 & 0.196 \\
\hline 19 & 1 & 19 & 19 & 2 & 18 & 45472.560 & 0.068 & 0.050 & 0.5 & 5.869 & 0.149 \\
\hline 19 & 2 & 18 & 20 & 1 & 19 & 45979.360 & -0.175 & 0.050 & -1.4 & -126.585 & 0.176 \\
\hline 20 & 0 & 20 & 20 & 1 & 19 & 21949.660 & 0.064 & 0.050 & 0.5 & -41.778 & 0.270 \\
\hline 20 & 1 & 20 & 20 & 2 & 19 & 46218.370 & 0.090 & 0.050 & 0.7 & 3.081 & 0.202 \\
\hline 20 & 1 & 19 & 20 & 2 & 18 & 33311.660 & -0.021 & 0.050 & -0.2 & 58.336 & -0.075 \\
\hline 20 & 2 & 19 & 21 & 1 & 20 & 50518.120 & -0.207 & 0.050 & -1.7 & -146.496 & 0.231 \\
\hline 21 & 4 & 18 & 20 & 5 & 15 & 34201.570 & -0.022 & 0.050 & 9.9 & 41.367 & $-6.5 E-3$ \\
\hline 21 & 4 & 17 & 20 & 5 & 16 & 34199.760 & 0.049 & 0.050 & 9.9 & 41.421 & $-6.7 E-3$ \\
\hline 21 & 0 & 21 & 21 & 1 & 20 & 23003.700 & 0.098 & 0.050 & 0.8 & -53.749 & 0.367 \\
\hline 21 & 1 & 21 & 21 & 2 & 20 & 47002.090 & 0.098 & 0.050 & 0.8 & -0.673 & 0.269 \\
\hline 21 & 1 & 20 & 21 & 2 & 19 & 33046.690 & 0.065 & 0.050 & 0.5 & 63.117 & -0.086 \\
\hline 21 & 2 & 19 & 22 & 1 & 22 & 34453.540 & -0.104 & 0.050 & -1.0 & -20.004 & -0.520 \\
\hline 22 & 0 & 22 & 22 & 1 & 21 & 24128.610 & 0.053 & 0.050 & 0.4 & -67.966 & 0.491 \\
\hline 22 & 1 & 22 & 22 & 2 & 21 & 47823.540 & 0.030 & 0.050 & 0.2 & -5.509 & 0.354 \\
\hline 22 & 1 & 21 & 22 & 2 & 20 & 32812.590 & 0.043 & 0.050 & 0.3 & 67.297 & -0.094 \\
\hline 22 & 2 & 20 & 23 & 1 & 23 & 36951.260 & 0.003 & 0.050 & 0.0 & -13.271 & -0.694 \\
\hline 22 & 3 & 20 & 23 & 2 & 21 & 27238.570 & -0.330 & 0.050 & -2.7 & -186.822 & 0.224 \\
\hline 22 & 3 & 19 & 23 & 2 & 22 & 23504.940 & -0.118 & 0.050 & -0.9 & -129.542 & -0.094 \\
\hline
\end{tabular}




\begin{tabular}{|c|c|c|c|c|c|c|c|c|c|c|c|}
\hline 23 & 0 & 23 & 23 & 1 & 22 & 25325.300 & 0.048 & 0.050 & 0.4 & -84.657 & 0.649 \\
\hline 23 & 1 & 23 & 23 & 2 & 22 & 48682.930 & 0.256 & 0.050 & 2.1 & -11.548 & 0.460 \\
\hline 23 & 1 & 22 & 23 & 2 & 21 & 32614.010 & 0.121 & 0.050 & 0.9 & 70.654 & -0.098 \\
\hline 23 & 3 & 21 & 24 & 2 & 22 & 31518.880 & -0.239 & 0.050 & -2.0 & -216.302 & 0.306 \\
\hline 24 & 0 & 24 & 24 & 1 & 23 & 26594.130 & 0.082 & 0.050 & 0.7 & -104.036 & 0.848 \\
\hline 24 & 1 & 24 & 24 & 2 & 23 & 49579.480 & 0.209 & 0.050 & 1.7 & -18.912 & 0.592 \\
\hline 24 & 1 & 23 & 24 & 2 & 22 & 32455.080 & 0.158 & 0.050 & 1.2 & 72.946 & -0.093 \\
\hline 24 & 3 & 22 & 25 & 2 & 23 & 35842.170 & -0.371 & 0.050 & -3.3 & -248.848 & 0.410 \\
\hline 25 & 0 & 25 & 25 & 1 & 24 & 27934.910 & 0.063 & 0.050 & 0.5 & -126.309 & 1.094 \\
\hline 25 & 1 & 25 & 25 & 2 & 24 & 50513.030 & -0.007 & 0.050 & -0.1 & -27.727 & 0.753 \\
\hline 25 & 1 & 24 & 25 & 2 & 23 & 32339.850 & 0.138 & 0.050 & 1.0 & 73.914 & -0.078 \\
\hline 25 & 3 & 23 & 26 & 2 & 24 & 40209.720 & -0.319 & 0.050 & -3.0 & -284.657 & 0.543 \\
\hline 25 & 3 & 22 & 26 & 2 & 25 & 34307.590 & -0.087 & 0.050 & -0.8 & -172.412 & -0.256 \\
\hline 26 & 0 & 26 & 26 & 1 & 25 & 29347.120 & 0.050 & 0.050 & 0.4 & -151.660 & 1.396 \\
\hline 26 & 1 & 25 & 26 & 2 & 24 & 32272.230 & 0.125 & 0.050 & 0.9 & 73.275 & -0.047 \\
\hline 26 & 3 & 24 & 27 & 2 & 25 & 44622.000 & -0.150 & 0.050 & -1.5 & -323.915 & 0.709 \\
\hline 26 & 3 & 23 & 27 & 2 & 26 & 37838.960 & 0.021 & 0.050 & 0.2 & -186.244 & -0.348 \\
\hline 27 & 0 & 27 & 27 & 1 & 26 & 30829.630 & -0.024 & 0.050 & -0.2 & -180.253 & 1.764 \\
\hline 27 & 1 & 26 & 27 & 2 & 25 & 32255.860 & 0.144 & 0.050 & 1.1 & 70.730 & $4.7 E-3$ \\
\hline 27 & 2 & 25 & 28 & 1 & 28 & 47911.630 & 0.004 & 0.050 & 9.9 & 66.316 & -2.512 \\
\hline 27 & 3 & 25 & 28 & 2 & 26 & 49078.780 & -0.296 & 0.050 & -3.3 & -366.801 & 0.914 \\
\hline 27 & 3 & 24 & 28 & 2 & 27 & 41330.310 & 0.035 & 0.050 & 0.4 & -199.426 & -0.469 \\
\hline 28 & 0 & 28 & 28 & 1 & 27 & 32381.150 & 0.100 & 0.050 & 0.9 & -212.221 & 2.207 \\
\hline 28 & 1 & 27 & 28 & 2 & 26 & 32293.940 & 0.013 & 0.050 & 0.1 & 65.962 & 0.084 \\
\hline 29 & 0 & 29 & 29 & 1 & 28 & 33999.270 & 0.032 & 0.050 & 0.3 & -247.668 & 2.735 \\
\hline 29 & 1 & 28 & 29 & 2 & 27 & 32390.060 & 0.170 & 0.050 & 1.2 & 58.634 & 0.198 \\
\hline 29 & 4 & 26 & 30 & 3 & 27 & 26992.940 & -0.018 & 0.050 & -0.2 & -367.347 & 0.371 \\
\hline 29 & 4 & 25 & 30 & 3 & 28 & 26135.870 & 0.113 & 0.050 & 1.2 & -332.151 & 0.050 \\
\hline 30 & 0 & 30 & 30 & 1 & 29 & 35681.800 & 0.050 & 0.050 & 0.5 & -286.662 & 3.359 \\
\hline 30 & 1 & 29 & 30 & 2 & 28 & 32546.680 & 0.146 & 0.050 & 1.1 & 48.391 & 0.356 \\
\hline 30 & 4 & 27 & 31 & 3 & 28 & 31009.580 & 0.430 & 0.050 & 6.3 & -410.811 & 0.491 \\
\hline 30 & 4 & 26 & 31 & 3 & 29 & 29974.020 & 0.184 & 0.050 & 2.4 & -365.631 & 0.051 \\
\hline 31 & 0 & 31 & 31 & 1 & 30 & 37425.790 & 0.087 & 0.050 & 1.0 & -329.237 & 4.092 \\
\hline 31 & 1 & 30 & 31 & 2 & 29 & 32766.700 & 0.127 & 0.050 & 0.9 & 34.861 & 0.568 \\
\hline 31 & 4 & 28 & 32 & 3 & 29 & 35047.710 & 0.299 & 0.050 & 8.2 & -457.903 & 0.645 \\
\hline 32 & 0 & 32 & 32 & 1 & 31 & 39227.840 & 0.004 & 0.050 & 0.1 & -375.386 & 4.945 \\
\hline 32 & 1 & 31 & 32 & 2 & 30 & 33052.660 & 0.135 & 0.050 & 1.0 & 17.652 & 0.847 \\
\hline 33 & 1 & 32 & 33 & 2 & 31 & 33406.820 & 0.107 & 0.050 & 0.8 & -3.643 & 1.206 \\
\hline 34 & 1 & 33 & 34 & 2 & 32 & 33831.390 & 0.112 & 0.050 & 0.9 & -29.449 & 1.660 \\
\hline 34 & 1 & 33 & 34 & 2 & 32 & 33831.390 & 0.112 & 0.050 & 0.9 & -29.449 & 1.660 \\
\hline 34 & 4 & 30 & 35 & 3 & 33 & 45247.070 & 0.171 & 0.050 & 9.9 & -511.634 & -0.011 \\
\hline 35 & 1 & 34 & 35 & 2 & 33 & 34328.260 & 0.080 & 0.050 & 0.7 & -60.202 & 2.229 \\
\hline 36 & 1 & 35 & 36 & 2 & 34 & 34899.260 & 0.063 & 0.050 & 0.5 & -96.347 & 2.930 \\
\hline 37 & 0 & 37 & 37 & 1 & 36 & 48976.450 & -0.068 & 0.050 & 9.9 & -656.946 & 11.439 \\
\hline 37 & 1 & 36 & 37 & 2 & 35 & 35545.980 & 0.061 & 0.050 & 0.5 & -138.336 & 3.788 \\
\hline 38 & 1 & 37 & 38 & 2 & 36 & 36269.820 & 0.084 & 0.050 & 0.8 & -186.617 & 4.825 \\
\hline 39 & 1 & 38 & 39 & 2 & 37 & 37071.840 & 0.020 & 0.050 & 0.2 & -241.631 & 6.069 \\
\hline 40 & 1 & 39 & 40 & 2 & 38 & 37953.130 & 0.024 & 0.050 & 0.3 & -303.801 & 7.549 \\
\hline 41 & 1 & 40 & 41 & 2 & 39 & 38914.320 & 0.052 & 0.050 & 0.6 & -373.523 & 9.296 \\
\hline 42 & 1 & 41 & 42 & 2 & 40 & 39955.700 & 0.008 & 0.050 & 0.1 & -451.156 & 11.343 \\
\hline 44 & 1 & 43 & 44 & 2 & 42 & 42279.180 & -0.121 & 0.050 & -1.5 & -631.348 & 16.477 \\
\hline 44 & 2 & 42 & 44 & 3 & 41 & 50743.500 & -0.006 & 0.050 & -0.1 & 592.034 & -5.536 \\
\hline 45 & 1 & 44 & 45 & 2 & 43 & 43560.730 & 0.115 & 0.050 & 1.5 & -734.354 & 19.638 \\
\hline 45 & 2 & 43 & 45 & 3 & 42 & 50368.620 & -0.041 & 0.050 & -0.7 & 588.191 & -5.488 \\
\hline 46 & 1 & 45 & 46 & 2 & 44 & 44920.550 & 0.134 & 0.050 & 2.1 & -846.146 & 23.246 \\
\hline 46 & 2 & 44 & 46 & 3 & 43 & 50043.620 & -0.107 & 0.050 & -1.5 & 577.228 & -5.266 \\
\hline 47 & 1 & 46 & 47 & 2 & 45 & 46357.120 & -0.219 & 0.050 & 9.9 & -966.759 & 27.340 \\
\hline 47 & 2 & 45 & 47 & 3 & 44 & 49772.620 & -0.172 & 0.050 & -2.3 & 558.409 & -4.830 \\
\hline 48 & 1 & 47 & 48 & 2 & 46 & 47869.510 & -0.126 & 0.050 & 9.9 & -1096.140 & 31.958 \\
\hline 48 & 2 & 46 & 48 & 3 & 45 & 49559.550 & -0.130 & 0.050 & -1.6 & 530.998 & -4.138 \\
\hline 49 & 1 & 48 & 49 & 2 & 47 & 49454.920 & -0.255 & 0.050 & 9.9 & -1234.150 & 37.138 \\
\hline 49 & 2 & 47 & 49 & 3 & 46 & 49407.790 & -0.153 & 0.050 & -1.9 & 494.260 & -3.143 \\
\hline 50 & 2 & 48 & 50 & 3 & 47 & 49320.730 & -0.128 & 0.050 & -1.5 & 447.465 & -1.794 \\
\hline
\end{tabular}




$\begin{array}{rrrrrrrrrrrr}51 & 2 & 49 & 51 & 3 & 48 & 49301.270 & -0.155 & 0.050 & -1.8 & 389.887 & -0.034 \\ 52 & 2 & 50 & 52 & 3 & 49 & 49352.210 & -0.161 & 0.050 & -1.8 & 320.807 & 2.195 \\ 53 & 2 & 51 & 53 & 3 & 50 & 49476.150 & -0.009 & 0.050 & -0.1 & 239.509 & 4.960 \\ 54 & 2 & 52 & 54 & 3 & 51 & 49674.960 & -0.028 & 0.050 & -0.3 & 145.290 & 8.332 \\ 55 & 2 & 53 & 55 & 3 & 52 & 49950.960 & 0.153 & 0.050 & 2.6 & 37.457 & 12.385 \\ 56 & 2 & 54 & 56 & 3 & 53 & 50305.480 & 0.160 & 0.050 & 9.9 & -84.667 & 17.200 \\ 57 & 2 & 55 & 57 & 3 & 54 & 50740.270 & 0.282 & 0.050 & 9.9 & -221.734 & 22.863\end{array}$

rms deviation: 2.7769 time (sec): 0.22

Gramdet.: $7.1 \mathrm{E}-7$

Rotational constants ( $\mathrm{MHz}$ ) and kappa:

$$
\begin{array}{rrrrr}
14751.0877 & 1967.79334 & 1895.38576 & -0.98873534 \\
+- & 0.0031 & 0.00058 & 0.00059 & 0.00000005
\end{array}
$$

Inertial constants and defect $(u \AA * * 2)$ :

$\begin{array}{rrrrr} & 34.2604618 & 256.825280 & 266.636527 & -24.44921 \\ +- & 0.0000073 & 0.000076 & 0.000083 & 0.00004\end{array}$

Quartic distortion constants:

$\begin{array}{rrrrrr} & 1.58674 & -40.8215 & 394.21 & 0.2292348 & 1.780 \\ +- & 0.00059 & 0.0087 & 0.23 & 0.000100 & 0.031\end{array}$

Standard distortion constants $(\mathrm{kHz})$ :

$\begin{array}{llllll}-1419.920 & -8.180850 & -4.513094 & 83.33215 & -6.346972 & 67.25990\end{array}$

Sextic and higher distortion constants:

\begin{tabular}{|c|c|c|c|c|c|}
\hline & 0 & 0 & 0 & 0 & 0.00263057 \\
\hline+- & fixed & fixed & fixed & fixed & 0.000035 \\
\hline+- & fixed & fixed & fixed & fixed & fixed \\
\hline
\end{tabular}

Significance estimate for fixed constants:

$\begin{array}{rlllllll}9: & 2.7258 \mathrm{E}-1 & 10: & 5.5843 \mathrm{E}-1 & 11: & 1.1853 \mathrm{E}-4 & 12: & 1.5934 \mathrm{E}-1 \\ 14: & 3.0240 \mathrm{E}-2 & 15: & 1.8456 \mathrm{E}-1 & 16: & 1.4873 \mathrm{E}-2 & 17: & 5.5843 \mathrm{E}-1 \\ 18: & 1.5934 \mathrm{E}-1 & 19: & 1.8456 \mathrm{E}-1 & 20: & 5.5843 \mathrm{E}-1 & 21: & 1.8456 \mathrm{E}-1 \\ 22: & 1.8456 \mathrm{E}-1 & & & & & & \end{array}$

Uncorrelation estimate for fixed constants:

$\begin{array}{rlllllll}9: & 2.2570 \mathrm{E}-1 & 10: & 1.9026 \mathrm{E}-1 & 11: & 0.0000 \mathrm{E}-9 & 12: & 1.9969 \mathrm{E}-1 \\ 14: & 3.3437 \mathrm{E}-1 & 15: & 2.0318 \mathrm{E}-1 & 16: & 4.2580 \mathrm{E}-1 & 17: & 1.9026 \mathrm{E}-1 \\ 18: & 1.9969 \mathrm{E}-1 & 19: & 2.0318 \mathrm{E}-1 & 20: & 1.9026 \mathrm{E}-1 & 21: & 2.0318 \mathrm{E}-1 \\ 22: & 2.0318 \mathrm{E}-1 & & & & & & \end{array}$

Significant Digits and Correlation Matrix:

9

\begin{tabular}{|c|c|c|c|c|c|c|c|}
\hline \multirow{8}{*}{$\begin{array}{l}0.088 \\
9\end{array}$} & 0.277 & 0.064 & 0.140 & 0.374 & -0.335 & -0.059 & -0.278 \\
\hline & 0.880 & 0.526 & -0.011 & -0.185 & 0.200 & 0.058 & 0.184 \\
\hline & 9 & 0.565 & 0.127 & -0.132 & -0.236 & -0.122 & -0.232 \\
\hline & & 6 & 0.435 & 0.125 & 0.020 & -0.399 & -0.099 \\
\hline & & & 6 & -0.266 & -0.038 & -0.882 & -0.29 \\
\hline & & & & 5 & -0.123 & 0.176 & -0.04 \\
\hline & & & & & 7 & 0.115 & 0.926 \\
\hline & & & & & & 4 & 0.420 \\
\hline
\end{tabular}


TABLE 2S: Allylgermane-74 1st ex. C2-C3 tors. state

Calculation by ROTFIT-TPW-1.5 of 29-MAY-1995 (with weights)

Watson A-reduction:

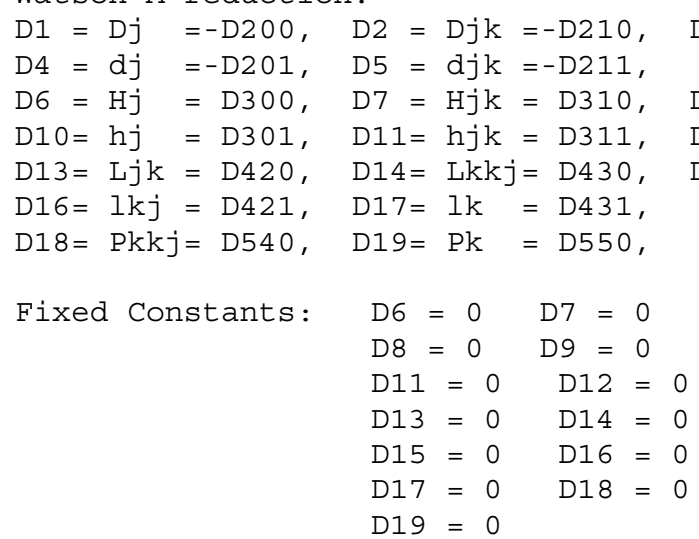

Total number: 104

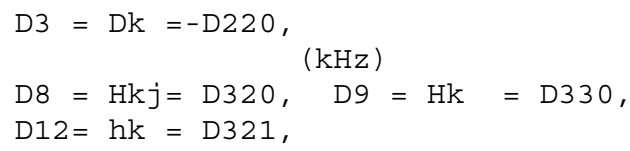

Transition obs. frequency

\begin{tabular}{|c|c|c|c|c|c|c|}
\hline 2 & 1 & 1 & 3 & 2 & 2 & 49939.410 \\
\hline 3 & 0 & 3 & 4 & 1 & 4 & 27938.220 \\
\hline 6 & 1 & 6 & 6 & 2 & 5 & 39234.340 \\
\hline 6 & 0 & 6 & 7 & 1 & 7 & 38894.440 \\
\hline 7 & 1 & 7 & 7 & 2 & 6 & 39507.660 \\
\hline 7 & 1 & 6 & 7 & 2 & 5 & 37392.420 \\
\hline 8 & 0 & 8 & 8 & 1 & 7 & 14253.910 \\
\hline 8 & 1 & 8 & 8 & 2 & 7 & 39820.360 \\
\hline 8 & 1 & 7 & 8 & 2 & 6 & 37118.120 \\
\hline 8 & 0 & 8 & 9 & 1 & 9 & 46028.520 \\
\hline 9 & 0 & 9 & 9 & 1 & 8 & 14632.800 \\
\hline 9 & 1 & 9 & 9 & 2 & 8 & 40172.790 \\
\hline 9 & 1 & 8 & 9 & 2 & 7 & 36819.180 \\
\hline 9 & 0 & 9 & 10 & 1 & 10 & 49549.480 \\
\hline 9 & 1 & 9 & 10 & 0 & 10 & 27560.710 \\
\hline 10 & 0 & 10 & 10 & 1 & 9 & 15061.210 \\
\hline 10 & 1 & 10 & 10 & 2 & 9 & 40564.830 \\
\hline 10 & 1 & 9 & 10 & 2 & 8 & 36499.350 \\
\hline 10 & 1 & 10 & 11 & 0 & 11 & 31759.440 \\
\hline 11 & 0 & 11 & 11 & 1 & 10 & 15541.530 \\
\hline 11 & 1 & 11 & 11 & 2 & 10 & 40996.890 \\
\hline 11 & 1 & 10 & 11 & 2 & 9 & 36161.940 \\
\hline 11 & 1 & 11 & 12 & 0 & 12 & 35977.880 \\
\hline 12 & 0 & 12 & 12 & 1 & 11 & 16076.880 \\
\hline 12 & 1 & 12 & 12 & 2 & 11 & 41468.930 \\
\hline 12 & 1 & 11 & 12 & 2 & 10 & 35811.310 \\
\hline 12 & 1 & 12 & 13 & 0 & 13 & 40213.190 \\
\hline 13 & 0 & 13 & 13 & 1 & 12 & 16669.430 \\
\hline 13 & 1 & 13 & 13 & 2 & 12 & 41980.890 \\
\hline 13 & 1 & 12 & 13 & 2 & 11 & 35451.550 \\
\hline 13 & 1 & 13 & 14 & 0 & 14 & 44462.040 \\
\hline 14 & 0 & 14 & 14 & 1 & 13 & 17321.630 \\
\hline 14 & 1 & 13 & 14 & 2 & 12 & 35087.570 \\
\hline 14 & 1 & 14 & 15 & 0 & 15 & 48721.140 \\
\hline 15 & 1 & 15 & 15 & 2 & 14 & 43126.390 \\
\hline 15 & 1 & 14 & 15 & 2 & 13 & 34723.860 \\
\hline 15 & 2 & 14 & 16 & 1 & 15 & 28676.580 \\
\hline
\end{tabular}

obs. - weight $t$ calc.

$-0.0110 .050$

$-0.0690 .050$

$-0.1890 .050$

$-0.104 \quad 0.050$

$-0.1460 .050$

0.0270 .050

$-0.1920 .050$

$-0.1710 .050$

0.0230 .050

0.1430 .050

0.0760 .050

$-0.0590 .050$

$-0.0790 .050$

0.0110 .050

$-0.178 \quad 0.050$

0.0320 .050

$\begin{array}{rl}-0.077 & 0.050\end{array}$

$0.070 \quad 0.050$

$-0.0030 .050$

$-0.2560 .050$

0.0340 .050

0.0360 .050

$0.003 \quad 0.050$

$-0.1330 .050$

0.0860 .050

0.1120 .050

$0.080 \quad 0.050$

$-0.006 \quad 0.050$

$-0.120 \quad 0.050$

$0.023 \quad 0.050$

$0.124 \quad 0.050$

$\begin{array}{lll}-0.071 & 0.050\end{array}$

0.0550 .050

$0.177 \quad 0.050$

0.0250 .050

$-0.1530 .050$

$-0.2790 .050$
$-0.1$

$-0.8$

$-2.1$

$-1.7$

$-1.6$

0.3

$-2.0$

$-1.9$

0.3

3.0

0.8

$-0.7$

$-0.9$

0.3

$-3.2$

0.3

$-0.9$

0.8

$-0.1$

$-2.7$

0.4

0.4

0.0

$-1.4$

1.0

1.2

1.1

$-0.1$

$-1.3$

0.3

1.7

$-0.7$

0.6

3.1

0.3

$-1.7$

9.9 distortion corrections total higher fixed

$\begin{array}{rr}-4.437 & -6.1 \mathrm{E}-7 \\ 0.153 & -2.3 \mathrm{E}-5 \\ -1.496 & 2.1 \mathrm{E}-4 \\ 0.550 & -4.9 \mathrm{E}-4 \\ -0.196 & 5.0 \mathrm{E}-4 \\ 1.640 & -4.5 \mathrm{E}-4 \\ 0.836 & 1.1 \mathrm{E}-3 \\ 1.169 & 1.1 \mathrm{E}-3 \\ 4.025 & -9.3 \mathrm{E}-4 \\ 0.644 & -2.0 \mathrm{E}-3 \\ 0.662 & 2.3 \mathrm{E}-3 \\ 2.552 & 2.1 \mathrm{E}-3 \\ 6.795 & -1.8 \mathrm{E}-3 \\ 0.627 & -3.6 \mathrm{E}-3 \\ -11.566 & 1.5 \mathrm{E}-3 \\ 0.217 & 4.2 \mathrm{E}-3 \\ 3.896 & 3.8 \mathrm{E}-3 \\ 9.962 & -3.1 \mathrm{E}-3 \\ -15.239 & 2.6 \mathrm{E}-3 \\ -0.592 & 7.4 \mathrm{E}-3 \\ 5.141 & 6.6 \mathrm{E}-3 \\ 13.529 & -5.1 \mathrm{E}-3 \\ -19.576 & 4.3 \mathrm{E}-3 \\ -1.876 & 0.013 \\ 6.219 & 0.011 \\ 17.489 & -8.0 \mathrm{E}-3 \\ -24.614 & 6.8 \mathrm{E}-3 \\ -3.761 & 0.020 \\ 7.055 & 0.017 \\ 21.821 & -0.012 \\ -30.381 & 0.010 \\ -6.394 & 0.032 \\ 26.485 & -0.017 \\ -36.895 & 0.014 \\ 7.671 & 0.040 \\ 31.426 & -0.024 \\ -66.404 & 0.053\end{array}$




\begin{tabular}{|c|c|c|c|c|c|c|c|c|c|c|c|}
\hline 16 & 0 & 16 & 16 & 1 & 15 & 18816.270 & -0.100 & 0.050 & $-1 \cdot 1$ & -14.570 & 0.073 \\
\hline 16 & 1 & 16 & 16 & 2 & 15 & 43759.730 & -0.020 & 0.050 & -0.2 & 7.270 & 0.058 \\
\hline 16 & 1 & 15 & 16 & 2 & 14 & 34366.150 & 0.100 & 0.050 & 1.1 & 36.564 & -0.032 \\
\hline 16 & 2 & 15 & 17 & 1 & 16 & 33160.380 & 0.027 & 0.050 & 9.9 & -79.942 & 0.075 \\
\hline 17 & 0 & 17 & 17 & 1 & 16 & 19663.880 & -0.044 & 0.050 & -0.5 & -20.492 & 0.106 \\
\hline 17 & 1 & 17 & 17 & 2 & 16 & 44433.730 & 0.036 & 0.050 & 0.4 & 6.266 & 0.083 \\
\hline 18 & 0 & 18 & 18 & 1 & 17 & 20581.370 & -0.118 & 0.050 & $-1 \cdot 3$ & -27.916 & 0.152 \\
\hline 18 & 1 & 18 & 18 & 2 & 17 & 45148.310 & 0.088 & 0.050 & 1.0 & 4.553 & 0.117 \\
\hline 19 & 0 & 19 & 19 & 1 & 18 & 21571.140 & -0.044 & 0.050 & -0.5 & -37.066 & 0.213 \\
\hline 19 & 1 & 19 & 19 & 2 & 18 & 45903.430 & 0.108 & 0.050 & 1.2 & 2.021 & 0.160 \\
\hline 19 & 1 & 18 & 19 & 2 & 17 & 33377.080 & -0.165 & 0.050 & $-1 \cdot 8$ & 52.026 & -0.063 \\
\hline 20 & 0 & 20 & 20 & 1 & 19 & 22634.800 & -0.028 & 0.050 & -0.3 & -48.174 & 0.294 \\
\hline 20 & 1 & 20 & 20 & 2 & 19 & 46699.040 & 0.102 & 0.050 & 1.1 & -1.445 & 0.217 \\
\hline 20 & 1 & 19 & 20 & 2 & 18 & 33093.390 & -0.034 & 0.050 & -0.4 & 56.694 & -0.073 \\
\hline 21 & 1 & 21 & 21 & 2 & 20 & 47535.140 & 0.174 & 0.050 & 1.9 & -5.965 & 0.289 \\
\hline 21 & 1 & 20 & 21 & 2 & 19 & 32841.070 & -0.003 & 0.050 & -0.0 & 60.807 & -0.082 \\
\hline 22 & 0 & 22 & 22 & 1 & 21 & 24989.340 & 0.005 & 0.050 & 0.1 & -77.209 & 0.534 \\
\hline 22 & 1 & 22 & 22 & 2 & 21 & 48411.420 & 0.166 & 0.050 & 1.8 & -11.662 & 0.380 \\
\hline 22 & 1 & 21 & 22 & 2 & 20 & 32625.160 & 0.009 & 0.050 & 0.1 & 64.141 & -0.086 \\
\hline 23 & 0 & 23 & 23 & 1 & 22 & 26281.720 & -0.045 & 0.050 & -0.5 & -95.597 & 0.706 \\
\hline 23 & 1 & 23 & 23 & 2 & 22 & 49327.680 & 0.089 & 0.050 & 1.0 & -18.663 & 0.495 \\
\hline 23 & 1 & 22 & 23 & 2 & 21 & 32450.450 & 0.021 & 0.050 & 0.2 & 66.451 & -0.084 \\
\hline 24 & 0 & 24 & 24 & 1 & 23 & 27651.170 & -0.021 & 0.050 & -0.2 & -116.858 & 0.921 \\
\hline 24 & 1 & 24 & 24 & 2 & 23 & 50283.840 & 0.131 & 0.050 & 1.5 & -27.095 & 0.636 \\
\hline 24 & 1 & 23 & 24 & 2 & 22 & 32321.490 & 0.034 & 0.050 & 0.4 & 67.469 & -0.072 \\
\hline 25 & 0 & 25 & 25 & 1 & 24 & 29097.050 & -0.051 & 0.050 & -0.6 & -141.186 & 1.187 \\
\hline 25 & 1 & 24 & 25 & 2 & 23 & 32242.610 & 0.074 & 0.050 & 0.8 & 66.905 & -0.047 \\
\hline 26 & 0 & 26 & 26 & 1 & 25 & 30618.280 & -0.150 & 0.050 & -1.7 & -168.754 & 1.514 \\
\hline 26 & 1 & 25 & 26 & 2 & 24 & 32217.760 & 0.047 & 0.050 & 0.5 & 64.450 & $-2.5 E-3$ \\
\hline 27 & 0 & 27 & 27 & 1 & 26 & 32213.610 & 0.054 & 0.050 & 0.6 & -199.703 & 1.910 \\
\hline 27 & 1 & 26 & 27 & 2 & 25 & 32250.770 & 0.002 & 0.050 & 0.0 & 59.776 & 0.067 \\
\hline 28 & 1 & 27 & 28 & 2 & 26 & 32345.220 & 0.003 & 0.050 & 0.0 & 52.533 & 0.168 \\
\hline 29 & 0 & 29 & 29 & 1 & 28 & 35615.990 & -0.057 & 0.050 & -0.7 & -272.149 & 2.952 \\
\hline 29 & 1 & 28 & 29 & 2 & 27 & 32504.340 & 0.015 & 0.050 & 0.2 & 42.354 & 0.311 \\
\hline 30 & 0 & 30 & 30 & 1 & 29 & 37417.670 & 0.085 & 0.050 & 1.0 & -313.750 & 3.619 \\
\hline 30 & 1 & 29 & 30 & 2 & 28 & 32731.060 & -0.057 & 0.050 & -0.6 & 28.848 & 0.504 \\
\hline 31 & 0 & 31 & 31 & 1 & 30 & 39281.460 & 0.113 & 0.050 & 1.4 & -358.940 & 4.400 \\
\hline 31 & 1 & 30 & 31 & 2 & 29 & 33028.360 & -0.028 & 0.050 & -0.3 & 11.609 & 0.761 \\
\hline 32 & 0 & 32 & 32 & 1 & 31 & 41203.370 & 0.005 & 0.050 & 0.1 & -407.669 & 5.305 \\
\hline 32 & 1 & 31 & 32 & 2 & 30 & 33398.710 & -0.007 & 0.050 & -0.1 & -9.791 & 1.093 \\
\hline 33 & 0 & 33 & 33 & 1 & 32 & 43179.470 & 0.121 & 0.050 & 1.7 & -459.850 & 6.348 \\
\hline 33 & 1 & 32 & 33 & 2 & 31 & 33844.460 & -0.015 & 0.050 & -0.2 & -35.794 & 1.517 \\
\hline 34 & 0 & 34 & 34 & 1 & 33 & 45204.860 & 0.115 & 0.050 & 2.1 & -515.354 & 7.539 \\
\hline 34 & 1 & 33 & 34 & 2 & 32 & 34367.790 & -0.041 & 0.050 & -0.4 & -66.857 & 2.050 \\
\hline 35 & 1 & 34 & 35 & 2 & 33 & 34970.780 & 0.037 & 0.050 & 0.4 & -103.447 & 2.712 \\
\hline 36 & 0 & 36 & 36 & 1 & 35 & 49384.370 & -0.259 & 0.050 & 9.9 & -635.653 & 10.419 \\
\hline 37 & 1 & 36 & 37 & 2 & 35 & 36421.970 & -0.018 & 0.050 & -0.2 & -195.086 & 4.513 \\
\hline 38 & 1 & 37 & 38 & 2 & 36 & 37273.150 & 0.055 & 0.050 & 0.7 & -251.059 & 5.702 \\
\hline 39 & 1 & 38 & 39 & 2 & 37 & 38209.270 & 0.006 & 0.050 & 0.1 & -314.386 & 7.121 \\
\hline 40 & 1 & 39 & 40 & 2 & 38 & 39231.100 & -0.071 & 0.050 & -0.9 & -385.472 & 8.802 \\
\hline 41 & 1 & 40 & 41 & 2 & 39 & 40339.210 & 0.053 & 0.050 & 0.7 & -464.673 & 10.776 \\
\hline 42 & 1 & 41 & 42 & 2 & 40 & 41533.150 & -0.048 & 0.050 & -0.6 & -552.296 & 13.079 \\
\hline 43 & 1 & 42 & 43 & 2 & 41 & 42812.890 & 0.013 & 0.050 & 0.2 & -648.578 & 15.746 \\
\hline 43 & 2 & 41 & 43 & 3 & 40 & 50485.000 & 0.037 & 0.050 & 0.7 & 546.814 & -4.864 \\
\hline 44 & 1 & 43 & 44 & 2 & 42 & 44177.360 & 0.002 & 0.050 & 0.0 & -753.682 & 18.814 \\
\hline 44 & 2 & 42 & 44 & 3 & 41 & 50120.390 & -0.165 & 0.050 & -2.7 & 539.129 & -4.746 \\
\hline 45 & 1 & 44 & 45 & 2 & 43 & 45625.360 & -0.018 & 0.050 & -0.3 & -867.688 & 22.321 \\
\hline 45 & 2 & 43 & 45 & 3 & 42 & 49811.790 & -0.035 & 0.050 & -0.5 & 523.841 & -4.445 \\
\hline 47 & 2 & 45 & 47 & 3 & 44 & 49378.650 & -0.061 & 0.050 & -0.8 & 467.368 & -3.132 \\
\hline 48 & 1 & 47 & 48 & 2 & 46 & 50451.310 & -0.077 & 0.050 & 9.9 & -1262.516 & 35.847 \\
\hline 48 & 2 & 46 & 48 & 3 & 45 & 49262.140 & -0.074 & 0.050 & -1.0 & 424.646 & -2.027 \\
\hline 49 & 2 & 47 & 49 & 3 & 46 & 49217.160 & 0.000 & 0.050 & 0.0 & 371.246 & -0.556 \\
\hline 50 & 2 & 48 & 50 & 3 & 47 & 49246.690 & -0.016 & 0.050 & -0.2 & 306.410 & 1.341 \\
\hline
\end{tabular}




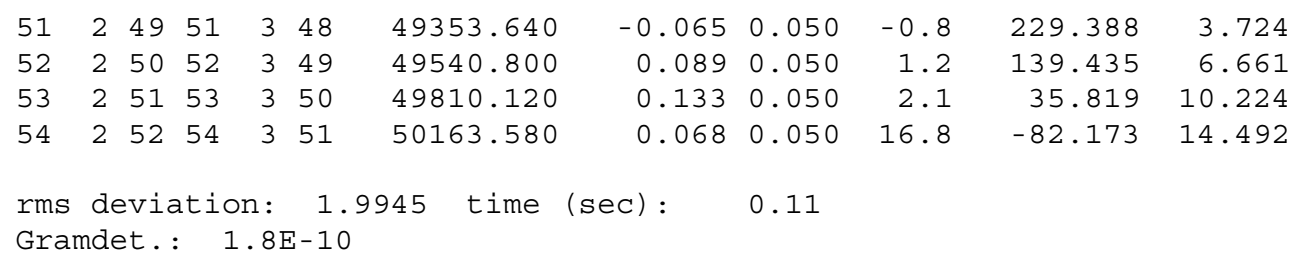

Sextic and higher distortion constants:

\begin{tabular}{|c|c|c|c|c|c|}
\hline & 0 & 0 & 0 & 0 & 0.00282308 \\
\hline+- & fixed & fixed & fixed & fixed & 0.000039 \\
\hline+- & fixed & fixed & fixed & fixed & fixed \\
\hline
\end{tabular}

Significance estimate for fixed constants:

$\begin{array}{rlllllll}9: & 2.7313 \mathrm{E}-2 & 10: & 1.6898 \mathrm{E}-2 & 11: & 4.1603 \mathrm{E}-6 & 12: & 6.1069 \mathrm{E}-3 \\ 14: & 4.5683 \mathrm{E}-3 & 15: & 2.2155 \mathrm{E}-4 & 16: & 1.1543 \mathrm{E}-4 & 17: & 1.6898 \mathrm{E}-2 \\ 18: & 6.1069 \mathrm{E}-3 & 19: & 2.2155 \mathrm{E}-4 & 20: & 1.6898 \mathrm{E}-2 & 21: & 2.2155 \mathrm{E}-4 \\ 22: & 2.2155 \mathrm{E}-4 & & & & & & \end{array}$

Uncorrelation estimate for fixed constants:

$\begin{array}{rlllllll}9: & 1.0088 \mathrm{E}-1 & 10: & 9.0646 \mathrm{E}-2 & 11: & 0.0000 \mathrm{E}-9 & 12: & 1.5240 \mathrm{E}-1 \\ 14: & 1.4795 \mathrm{E}-1 & 15: & 1.6997 \mathrm{E}-1 & 16: & 2.0307 \mathrm{E}-1 & 17: & 9.0646 \mathrm{E}-2 \\ 18: & 1.5240 \mathrm{E}-1 & 19: & 1.6997 \mathrm{E}-1 & 20: & 9.0646 \mathrm{E}-2 & 21: & 1.6997 \mathrm{E}-1 \\ 22: & 1.6997 \mathrm{E}-1 & & & & & & \end{array}$

Significant Digits and Correlation Matrix:

8

\begin{tabular}{|c|c|c|c|c|c|c|c|}
\hline 0.079 & 0.108 & -0.058 & -0.828 & 0.941 & -0.138 & 0.461 & 0.102 \\
\hline \multirow[t]{8}{*}{10} & 0.996 & 0.926 & 0.028 & -0.041 & 0.117 & -0.043 & 0.076 \\
\hline & 10 & 0.915 & 0.009 & -0.017 & 0.032 & -0.040 & 0.005 \\
\hline & & 5 & 0.131 & -0.148 & 0.160 & -0.130 & 0.072 \\
\hline & & & 6 & -0.937 & 0.108 & -0.747 & -0.214 \\
\hline & & & & 5 & -0.116 & 0.535 & 0.139 \\
\hline & & & & & 7 & -0.033 & 0.893 \\
\hline & & & & & & 5 & 0.370 \\
\hline & & & & & & & 6 \\
\hline
\end{tabular}


TABLE 3S: Allylgermane-72 ground vib. state

Calculation by ROTFIT-TPW-1.5 of 29-MAY-1995 (with weights)

Watson A-reduction:

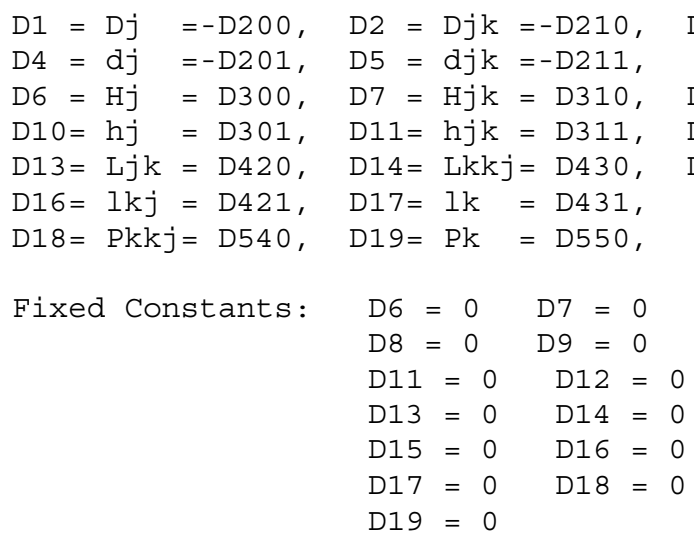

Total number: 131

Transition obs. frequency obs.- weight $t$ distortion corrections calc.

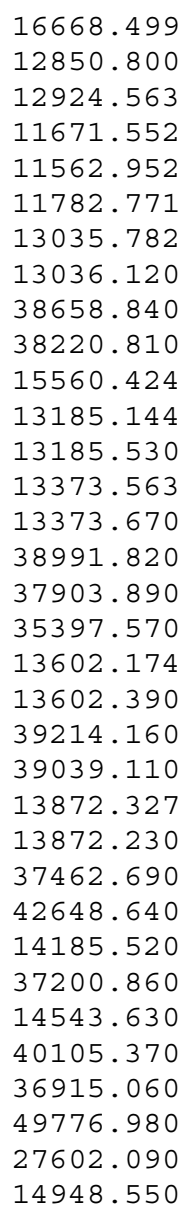

$\begin{array}{rlrrr}-0.008 & 0.0030 & 9.9 & -0.322 & -2.2 \mathrm{E}-8 \\ -0.008 & 0.0030 & 9.9 & -0.325 & 2.2 \mathrm{E}-8 \\ -0.007 & 0.0030 & 9.9 & -0.175 & 6.0 \mathrm{E}-7 \\ -0.001 & 0.0030 & 9.9 & -0.174 & -6.4 \mathrm{E}-8 \\ 0.002 & 0.0030 & 9.9 & 0.108 & -4.2 \mathrm{E}-6 \\ -0.000 & 0.0030 & 9.9 & 0.036 & 4.1 \mathrm{E}-6 \\ -0.006 & 0.0030 & -3.5 & 0.036 & 4.8 \mathrm{E}-6 \\ 0.332 & 0.050 & 2.3 & 0.036 & 4.8 \mathrm{E}-6 \\ -0.091 & 0.050 & -0.7 & -4.609 & 4.8 \mathrm{E}-6 \\ -0.015 & 0.050 & -0.1 & -4.500 & -4.7 \mathrm{E}-6 \\ -0.001 & 0.0030 & 9.9 & -0.412 & -5.0 \mathrm{E}-7 \\ -0.005 & 0.0030 & -2.2 & 0.289 & 2.2 \mathrm{E}-5 \\ 0.381 & 0.050 & 2.7 & 0.289 & 2.2 \mathrm{E}-5 \\ -0.004 & 0.0030 & -3.5 & 0.560 & 7.7 \mathrm{E}-5 \\ 0.103 & 0.050 & 0.7 & 0.560 & 7.7 \mathrm{E}-5 \\ -0.013 & 0.050 & -0.1 & -2.597 & 7.5 \mathrm{E}-5 \\ -0.016 & 0.050 & -0.1 & -2.083 & -7.1 \mathrm{E}-5 \\ 0.013 & 0.050 & 0.1 & 0.409 & -2.0 \mathrm{E}-4 \\ -0.003 & 0.0030 & 9.9 & 0.816 & 2.1 \mathrm{E}-4 \\ 0.213 & 0.050 & 1.5 & 0.816 & 2.1 \mathrm{E}-4 \\ 0.100 & 0.050 & 0.8 & -1.340 & 2.0 \mathrm{E}-4 \\ 0.086 & 0.050 & 0.6 & 0.498 & -4.8 \mathrm{E}-4 \\ -0.002 & 0.0030 & 9.9 & 1.016 & 5.1 \mathrm{E}-4 \\ -0.099 & 0.050 & -0.7 & 1.016 & 5.1 \mathrm{E}-4 \\ 0.062 & 0.050 & 0.5 & 1.628 & -4.4 \mathrm{E}-4 \\ 0.357 & 0.050 & 2.6 & 0.547 & -1.0 \mathrm{E}-3 \\ -0.063 & 0.050 & -0.4 & 1.110 & 1.1 \mathrm{E}-3 \\ 0.111 & 0.050 & 0.8 & 4.024 & -9.1 \mathrm{E}-4 \\ -0.066 & 0.050 & -0.5 & 1.036 & 2.2 \mathrm{E}-3 \\ 0.199 & 0.050 & 1.5 & 3.001 & 2.0 \mathrm{E}-3 \\ 0.133 & 0.050 & 1.0 & 6.806 & -1.7 \mathrm{E}-3 \\ 0.223 & 0.050 & 1.6 & 0.507 & -3.5 \mathrm{E}-3 \\ 0.204 & 0.050 & 1.5 & -11.378 & 1.5 \mathrm{E}-3 \\ -0.055 & 0.050 & -0.4 & 0.719 & 4.1 \mathrm{E}-3\end{array}$

$(\mathrm{kHz})$

$\mathrm{D} 8=\mathrm{Hkj}=\mathrm{D} 320, \mathrm{D} 9=\mathrm{Hk}=\mathrm{D} 330$,

$\mathrm{D} 12=\mathrm{hk}=\mathrm{D} 321$,

$\mathrm{D} 15=\mathrm{Lk}=\mathrm{D} 440$,

$(\mathrm{Hz})$. 


\begin{tabular}{|c|c|c|c|c|c|c|c|c|c|c|c|}
\hline 10 & 1 & 10 & 10 & 2 & 9 & 40477.670 & 0.227 & 0.050 & 1.7 & 4.484 & $3.7 \mathrm{E}-3$ \\
\hline 10 & 1 & 9 & 10 & 2 & 8 & 36608.300 & 0.065 & 0.050 & 0.5 & 9.988 & $-3.0 E-3$ \\
\hline 10 & 1 & 10 & 11 & 0 & 11 & 31799.250 & -0.018 & 0.050 & -0.1 & -15.001 & $2.6 E-3$ \\
\hline 11 & 0 & 11 & 11 & 1 & 10 & 15402.320 & -0.094 & 0.050 & -0.7 & 0.072 & 7. $2 E-3$ \\
\hline 11 & 1 & 11 & 11 & 2 & 10 & 40887.730 & 0.173 & 0.050 & 1.3 & 5.893 & $6.4 \mathrm{E}-3$ \\
\hline 11 & 1 & 10 & 11 & 2 & 9 & 36284.210 & 0.152 & 0.050 & 1.2 & 13.578 & $-5.0 E-3$ \\
\hline 11 & 1 & 11 & 12 & 0 & 12 & 36016.390 & 0.051 & 0.050 & 0.4 & -19.284 & $4 \cdot 3 E-3$ \\
\hline 2 & 0 & 12 & 12 & 1 & 11 & 15907.250 & -0.113 & 0.050 & -0.8 & -1.008 & 0.012 \\
\hline 12 & 1 & 12 & 12 & 2 & 11 & 41335.760 & 0.114 & 0.050 & 0.8 & 7.163 & 0.011 \\
\hline 12 & 1 & 11 & 12 & 2 & 10 & 35946.340 & 0.265 & 0.050 & 2.0 & 17.573 & $-7.9 E-3$ \\
\hline 12 & 1 & 12 & 13 & 0 & 13 & 40250.310 & 0.011 & 0.050 & 0.1 & -24.266 & $6.8 E-3$ \\
\hline 13 & 0 & 13 & 13 & 1 & 12 & 16465.750 & -0.052 & 0.050 & -0.4 & -2.639 & 0.020 \\
\hline 13 & 1 & 13 & 13 & 2 & 12 & 41822.000 & 0.161 & 0.050 & 1.2 & 8.222 & 0.017 \\
\hline 13 & 1 & 12 & 13 & 2 & 11 & 35598.420 & 0.178 & 0.050 & 1.4 & 21.956 & -0.012 \\
\hline 14 & 0 & 14 & 14 & 1 & 13 & 17080.120 & -0.036 & 0.050 & -0.3 & -4.956 & 0.031 \\
\hline 14 & 1 & 14 & 14 & 2 & 13 & 42346.270 & 0.018 & 0.050 & 0.1 & 8.994 & 0.026 \\
\hline 14 & 1 & 13 & 14 & 2 & 12 & 35244.960 & 0.200 & 0.050 & 1.5 & 26.698 & -0.017 \\
\hline 14 & 1 & 14 & 15 & 0 & 15 & 48757.010 & 0.009 & 0.050 & 0.1 & -36.449 & 0.015 \\
\hline 15 & 0 & 15 & 15 & 1 & 14 & 17752.830 & -0.051 & 0.050 & -0.4 & -8.113 & 0.047 \\
\hline 15 & 1 & 15 & 15 & 2 & 14 & 42909.040 & 0.050 & 0.050 & 0.4 & 9.395 & 0.039 \\
\hline 15 & 2 & 14 & 16 & 1 & 15 & 28600.850 & -0.140 & 0.050 & -1.1 & -65.357 & 0.052 \\
\hline 16 & 0 & 16 & 16 & 1 & 15 & 18486.320 & -0.095 & 0.050 & -0.7 & -12.278 & 0.070 \\
\hline 16 & 2 & 15 & 17 & 1 & 16 & 33067.640 & -0.096 & 0.050 & -0.8 & -78.659 & 0.073 \\
\hline 17 & 0 & 17 & 17 & 1 & 16 & 19283.090 & -0.042 & 0.050 & -0.3 & -17.639 & 0.102 \\
\hline 17 & 2 & 16 & 18 & 1 & 17 & 37562.860 & -0.056 & 0.050 & -0.5 & -93.558 & 0.101 \\
\hline 18 & 0 & 18 & 18 & 1 & 17 & 20145.120 & -0.155 & 0.050 & -1.2 & -24.396 & 0.146 \\
\hline 18 & 1 & 18 & 18 & 2 & 17 & 44828.200 & 0.343 & 0.050 & 2.6 & 7.452 & 0.113 \\
\hline 18 & 2 & 17 & 19 & 1 & 18 & 42085.410 & -0.075 & 0.050 & -0.7 & -110.147 & 0.137 \\
\hline 19 & 0 & 19 & 19 & 1 & 18 & 21074.930 & 0.025 & 0.050 & 0.2 & -32.763 & 0.205 \\
\hline 19 & 0 & 19 & 19 & 1 & 18 & 21074.930 & 0.025 & 0.050 & 0.2 & -32.763 & 0.205 \\
\hline 19 & 1 & 19 & 19 & 2 & 18 & 45544.570 & 0.105 & 0.050 & 0.8 & 5.420 & 0.156 \\
\hline 19 & 1 & 18 & 19 & 2 & 17 & 33552.230 & -0.107 & 0.050 & -0.8 & 53.389 & -0.065 \\
\hline 19 & 2 & 18 & 20 & 1 & 19 & 46634.360 & 0.056 & 0.050 & 0.7 & -128.516 & 0.183 \\
\hline 20 & 0 & 20 & 20 & 1 & 19 & 22073.850 & 0.013 & 0.050 & 0.1 & -42.964 & 0.283 \\
\hline 20 & 0 & 20 & 20 & 1 & 19 & 22073.850 & 0.013 & 0.050 & 0.1 & -42.964 & 0.283 \\
\hline 20 & 1 & 20 & 20 & 2 & 19 & 46299.660 & 0.125 & 0.050 & 1.0 & 2.516 & 0.211 \\
\hline 21 & 0 & 21 & 21 & 1 & 20 & 23143.640 & 0.057 & 0.050 & 0.5 & -55.229 & 0.384 \\
\hline 21 & 0 & 21 & 21 & 1 & 20 & 23143.640 & 0.057 & 0.050 & 0.5 & -55.229 & 0.384 \\
\hline 22 & 0 & 22 & 22 & 1 & 21 & 24285.330 & 0.035 & 0.050 & 0.3 & -69.788 & 0.515 \\
\hline 22 & 1 & 22 & 22 & 2 & 21 & 47925.000 & 0.305 & 0.050 & 2.4 & -6.374 & 0.370 \\
\hline 22 & 3 & 20 & 23 & 2 & 21 & 27963.400 & -0.070 & 0.050 & -0.7 & -189.780 & 0.237 \\
\hline 23 & 0 & 23 & 23 & 1 & 22 & 25499.370 & -0.351 & 0.050 & -3.0 & -86.869 & 0.680 \\
\hline 23 & 1 & 23 & 23 & 2 & 22 & 48794.750 & 0.258 & 0.050 & 2.1 & -12.601 & 0.481 \\
\hline 23 & 1 & 22 & 23 & 2 & 21 & 32574.480 & 0.134 & 0.050 & 1.0 & 70.575 & -0.098 \\
\hline 23 & 3 & 20 & 24 & 2 & 23 & 27794.310 & -0.011 & 0.050 & -0.1 & -145.197 & -0.141 \\
\hline 24 & 0 & 24 & 24 & 1 & 23 & 26787.200 & 0.035 & 0.050 & 0.3 & -106.690 & 0.888 \\
\hline 24 & 1 & 24 & 24 & 2 & 23 & 49701.960 & -0.194 & 0.050 & -1.6 & -20.182 & 0.618 \\
\hline 24 & 3 & 22 & 25 & 2 & 23 & 36641.570 & -0.095 & 0.050 & $-1 \cdot 3$ & -252.967 & 0.432 \\
\hline 24 & 3 & 21 & 25 & 2 & 24 & 31417.500 & 0.164 & 0.050 & 3.4 & -159.645 & -0.196 \\
\hline 25 & 1 & 24 & 25 & 2 & 23 & 32312.390 & 0.124 & 0.050 & 0.9 & 73.430 & -0.075 \\
\hline 26 & 0 & 26 & 26 & 1 & 25 & 29579.990 & 0.069 & 0.050 & 0.6 & -155.350 & 1.462 \\
\hline 26 & 1 & 25 & 26 & 2 & 24 & 32252.680 & 0.191 & 0.050 & 1.4 & 72.503 & -0.040 \\
\hline 26 & 3 & 24 & 27 & 2 & 25 & 45498.590 & 0.120 & 0.050 & 9.9 & -329.440 & 0.746 \\
\hline 27 & 0 & 27 & 27 & 1 & 26 & 31083.620 & 0.217 & 0.050 & 2.0 & -184.534 & 1.846 \\
\hline 27 & 1 & 26 & 27 & 2 & 25 & 32245.350 & 0.190 & 0.050 & 1.4 & 69.605 & 0.016 \\
\hline 27 & 3 & 25 & 28 & 2 & 26 & 49994.410 & -0.456 & 0.050 & 9.9 & -373.124 & 0.961 \\
\hline 28 & 0 & 28 & 28 & 1 & 27 & 32656.270 & 0.032 & 0.050 & 0.3 & -217.137 & 2.308 \\
\hline 28 & 1 & 27 & 28 & 2 & 26 & 32293.940 & 0.254 & 0.050 & 1.8 & 64.409 & 0.102 \\
\hline 29 & 0 & 29 & 29 & 1 & 28 & 34296.330 & 0.042 & 0.050 & 0.4 & -253.259 & 2.860 \\
\hline 29 & 1 & 28 & 29 & 2 & 27 & 32401.390 & 0.152 & 0.050 & 1.1 & 56.573 & 0.225 \\
\hline 29 & 4 & 26 & 30 & 3 & 27 & 27893.350 & 0.115 & 0.050 & 9.9 & -372.707 & 0.395 \\
\hline 29 & 4 & 25 & 30 & 3 & 28 & 27004.240 & 0.192 & 0.050 & 9.9 & -335.976 & 0.051 \\
\hline 30 & 1 & 29 & 30 & 2 & 28 & 32570.840 & 0.079 & 0.050 & 0.6 & 45.732 & 0.395 \\
\hline
\end{tabular}




\begin{tabular}{|c|c|c|c|c|c|c|c|c|c|c|c|}
\hline 31 & 0 & 31 & 31 & 1 & 30 & 37767.290 & 0.055 & 0.050 & 0.8 & -336.274 & 4.27 \\
\hline 31 & 1 & 30 & 31 & 2 & 29 & 32805.090 & 0.105 & 0.050 & 0.8 & 31.505 & 0.62 \\
\hline & 1 & 31 & 32 & 2 & 30 & 33106.560 & 0.126 & 0.050 & 0.9 & 13.493 & \\
\hline 3 & 0 & 33 & 33 & 1 & 32 & 41470.750 & 0.043 & 0.050 & 9.9 & -433.625 & \\
\hline 3 & 1 & 32 & 33 & 2 & 31 & 3477.540 & 0.099 & 0.050 & 0.8 & -8.721 & 1.3 \\
\hline 4 & 1 & 33 & 34 & 2 & 32 & 33920.230 & 0.080 & 0.050 & 0.6 & -35.570 & \\
\hline 5 & 1 & 34 & 35 & 2 & 33 & 4436.570 & 0.050 & 0.050 & 0.4 & -67.498 & 2.3 \\
\hline & 1 & 35 & 36 & 2 & 34 & 5028.410 & 0.085 & 0.050 & 0.7 & -104.960 & \\
\hline 7 & 0 & 37 & 37 & 1 & 36 & 49448.140 & -0.200 & 0.050 & 9.9 & -668.496 & 1.9 \\
\hline 37 & 1 & 36 & 37 & 2 & 35 & 35697.180 & 0.039 & 0.050 & 0.4 & -148.411 & 4.0 \\
\hline 8 & 1 & 37 & 38 & 2 & 36 & 36444.420 & 0.084 & 0.050 & 0.9 & -198.306 & 5.1 \\
\hline 9 & 1 & 38 & 39 & 2 & 37 & 37271.130 & 0.080 & 0.050 & 0.9 & -255.087 & \\
\hline 0 & 1 & 39 & 40 & 2 & 38 & 38178.210 & 0.034 & 0.050 & 0.4 & -319.177 & 8.0 \\
\hline 1 & 1 & 40 & 41 & 2 & 39 & 39166.320 & -0.009 & 0.050 & -0.1 & -390.968 & 9.8 \\
\hline 2 & 1 & 41 & 42 & 2 & 40 & 40235.890 & 0.063 & 0.050 & 0.9 & -470.813 & 2.0 \\
\hline 43 & 1 & 42 & 43 & 2 & 41 & 41386.590 & -0.072 & 0.050 & -1.1 & -559.015 & 14.5 \\
\hline 4 & 2 & 42 & 44 & 3 & 41 & 50609.860 & -0.054 & 0.050 & 9.9 & 587.860 & -5.5 \\
\hline 5 & 1 & 44 & 45 & 2 & 43 & 43930.450 & -0.093 & 0.050 & -1.7 & -761.383 & 20.7 \\
\hline 45 & 2 & 43 & 45 & 3 & 42 & 50246.750 & 0.013 & 0.050 & 0.9 & 582.051 & -5.4 \\
\hline 46 & 1 & 45 & 46 & 2 & 44 & 45321.840 & 0.088 & 0.050 & 3.4 & -875.815 & 24.5 \\
\hline 6 & 2 & 44 & 46 & 3 & 43 & 49935.370 & 0.028 & 0.050 & 0.6 & 568.855 & -5.1 \\
\hline 7 & 2 & 45 & 47 & 3 & 44 & 49679.700 & -0.119 & 0.050 & -1.9 & 547.526 & -4.6 \\
\hline$B$ & 1 & 47 & 48 & 2 & 46 & 48335.070 & -0.124 & 0.050 & 9.9 & -1131.220 & 33.6 \\
\hline 48 & 2 & 46 & 48 & 3 & 45 & 49483.950 & -0.036 & 0.050 & -0.5 & 517.319 & -3.8 \\
\hline 19 & 1 & 48 & 49 & 2 & 47 & 49953.180 & -0.061 & 0.050 & 9.9 & -1271.942 & 39.1 \\
\hline 9 & 2 & 47 & 49 & 3 & 46 & 49351.140 & -0.243 & 0.050 & -3.2 & 477.489 & -2.7 \\
\hline 50 & 2 & 48 & 50 & 3 & 47 & 49285.190 & -0.073 & 0.050 & -0.9 & 427.300 & -1.2 \\
\hline 51 & 2 & 49 & 51 & 3 & 48 & 49288.500 & -0.098 & 0.050 & -1.2 & 366.016 & 0.6 \\
\hline 52 & 2 & 50 & 52 & 3 & 49 & 49364.050 & -0.034 & 0.050 & -0.4 & 292.910 & 3.0 \\
\hline 53 & 2 & 51 & 53 & 3 & 50 & 49514.030 & -0.114 & 0.050 & $-1 \cdot 3$ & 207.262 & 6.0 \\
\hline 54 & 2 & 52 & 54 & 3 & 51 & 49740.960 & 0.023 & 0.050 & 0.3 & 108.361 & 9.7 \\
\hline 5 & 2 & 53 & 55 & 3 & 52 & 50046.320 & -0.047 & 0.050 & -1.1 & -4.490 & 14.0 \\
\hline 56 & 2 & 54 & 56 & 3 & 53 & 50432.180 & 0.093 & 0.050 & 9.9 & -131.967 & 19.2 \\
\hline & 2 & 55 & 57 & 3 & 54 & 50899.660 & 0.156 & 0.050 & 9.9 & -274.721 & 25.3 \\
\hline
\end{tabular}

rms deviation: 2.8532 time (sec): 0.17

Gramdet.: $1.4 \mathrm{E}-7$

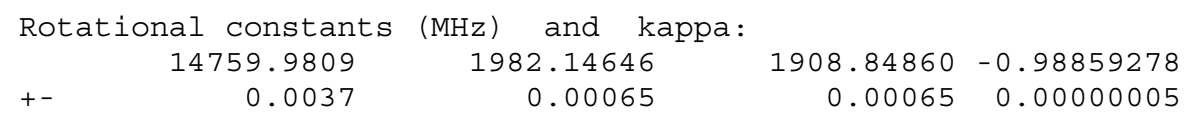

Inertial constants and defect ( $\mathrm{A} \star * 2)$ : $\begin{array}{llll}34.2398192 & 254.965557 & 264.755977 & -24.44940\end{array}$

$\begin{array}{lllll}+- & 0.0000086 & 0.000083 & 0.000091 & 0.00004\end{array}$

Quartic distortion constants:

$\begin{array}{cccccc} & 1.61966 & -41.1384 & 402.32 & 0.2338389 & 1.765 \\ +- & 0.0016 & 0.013 & 1.4 & 0.00010 & 0.035\end{array}$

Standard distortion constants $(\mathrm{kHz})$ :

$\begin{array}{llllll}-1451.195 & -8.349346 & -4.607923 & 83.79157 & -6.478635 & 67.80482\end{array}$

Sextic and higher distortion constants:

\begin{tabular}{|c|c|c|c|c|c|}
\hline & 0 & 0 & 0 & 0 & 0.00274750 \\
\hline+- & fixed & fixed & fixed & fixed & 0.000036 \\
\hline+- & fixed & fixed & fixed & fixed & fixed \\
\hline
\end{tabular}


Significance estimate for fixed constants:

$\begin{array}{rlllllll}9: & 1.5127 \mathrm{E}-2 & 10: & 3.6927 \mathrm{E}-1 & 11: & 3.6099 \mathrm{E}-5 & 12: & 2.2456 \mathrm{E}-1 \\ 14: & 2.3344 \mathrm{E}-1 & 15: & 1.7344 \mathrm{E}-1 & 16: & 2.0786 \mathrm{E}-1 & 17: & 3.6927 \mathrm{E}-1 \\ 18: & 2.2456 \mathrm{E}-1 & 19: & 1.7344 \mathrm{E}-1 & 20: & 3.6927 \mathrm{E}-1 & 21: & 1.7344 \mathrm{E}-1 \\ 22: & 1.7344 \mathrm{E}-1 & & & & & & \end{array}$

Uncorrelation estimate for fixed constants:

$\begin{array}{rlllllll}9: & 1.5534 \mathrm{E}-1 & 10: & 1.4953 \mathrm{E}-1 & 11: & 0.0000 \mathrm{E}-9 & 12: & 1.7261 \mathrm{E}-1 \\ 14: & 3.1534 \mathrm{E}-1 & 15: & 1.8290 \mathrm{E}-1 & 16: & 3.4089 \mathrm{E}-1 & 17: & 1.4953 \mathrm{E}-1 \\ 18: & 1.7261 \mathrm{E}-1 & 19: & 1.8290 \mathrm{E}-1 & 20: & 1.4953 \mathrm{E}-1 & 21: & 1.8290 \mathrm{E}-1 \\ 22: & 1.8290 \mathrm{E}-1 & & & & & & \end{array}$

Significant Digits and Correlation Matrix:

9

\begin{tabular}{|c|c|c|c|c|c|c|c|}
\hline \multirow{8}{*}{$\begin{array}{l}0.137 \\
9\end{array}$} & 0.307 & 0.303 & -0.200 & 0.457 & -0.328 & 0.045 & -0.239 \\
\hline & 0.888 & 0.438 & -0.143 & 0.183 & 0.192 & 0.067 & 0.174 \\
\hline & 9 & 0.404 & -0.043 & 0.161 & -0.227 & -0.071 & -0.211 \\
\hline & & 6 & -0.315 & 0.817 & 0.069 & -0.162 & -0.032 \\
\hline & & & 6 & -0.644 & -0.011 & -0.764 & -0.260 \\
\hline & & & & 5 & 0.000 & 0.126 & 0.019 \\
\hline & & & & & 7 & 0.066 & 0.899 \\
\hline & & & & & & 4 & 0.432 \\
\hline
\end{tabular}


TABLE 4S: Allylgermane-72 1st ex. C2-C3 tors. state

Calculation by ROTFIT-TPW-1.5 of 29-MAY-1995 (with weights)

Watson A-reduction:

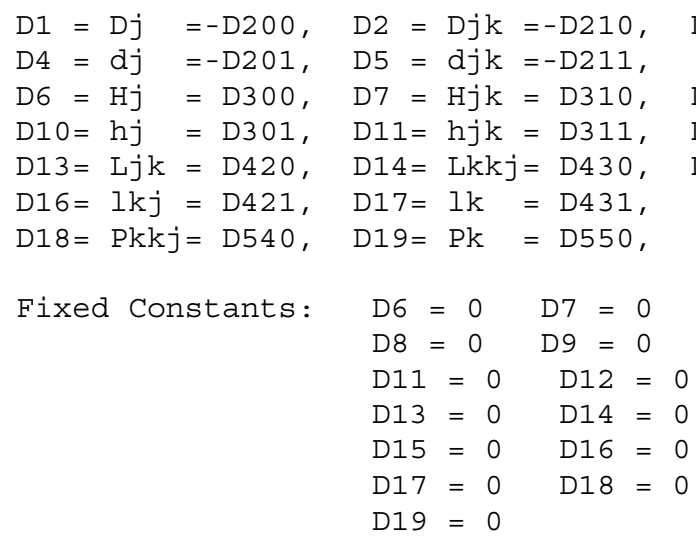

Total number: 87

\begin{tabular}{|c|c|c|c|c|c|c|c|c|c|c|c|}
\hline \multicolumn{4}{|c|}{ Transition } & \multicolumn{2}{|c|}{ obs. frequency } & $\begin{array}{l}\text { obs. - } \\
\text { calc. }\end{array}$ & weight & t & \multicolumn{3}{|c|}{ distortion corrections } \\
\hline 0 & 3 & 4 & 1 & 4 & 28039.680 & -0.154 & 0.050 & -1.9 & 0.161 & $-2.4 E-5$ & \\
\hline 0 & 5 & 6 & 1 & 6 & 35430.160 & -0.118 & 0.050 & -1.8 & 0.483 & $-2.2 E-4$ & \\
\hline 1 & 6 & 6 & 2 & 5 & 39228.700 & -0.028 & 0.050 & -0.3 & -1.575 & $2.2 \mathrm{E}-4$ & \\
\hline 1 & 5 & 6 & 2 & 4 & 37613.810 & -0.053 & 0.050 & -0.6 & -0.438 & $-2.0 E-4$ & \\
\hline 0 & 6 & 7 & 1 & 7 & 39071.550 & -0.016 & 0.050 & -0.3 & 0.610 & $-5.1 E-4$ & \\
\hline 1 & 7 & 7 & 2 & 6 & 39505.340 & -0.062 & 0.050 & -0.8 & -0.270 & $5.2 \mathrm{E}-4$ & \\
\hline 1 & 7 & 8 & 0 & 8 & 19475.120 & 0.093 & 0.050 & 2.3 & -5.999 & $4.1 E-4$ & \\
\hline 1 & 7 & 8 & 2 & 6 & 37087.130 & 0.045 & 0.050 & 0.5 & 4.009 & $-9.7 E-4$ & \\
\hline 0 & 8 & 9 & 1 & 9 & 46254.070 & 0.128 & 0.050 & 2.3 & 0.775 & $-2.1 E-3$ & \\
\hline 1 & 9 & 9 & 2 & 8 & 40178.810 & 0.079 & 0.050 & 1.0 & 2.484 & $2.2 E-3$ & \\
\hline 1 & 8 & 9 & 2 & 7 & 36785.230 & 0.100 & 0.050 & 1.2 & 6.805 & $-1.8 E-3$ & \\
\hline 0 & 9 & 10 & 1 & 10 & 49798.870 & -0.040 & 0.050 & -0.8 & 0.806 & $-3.8 E-3$ & \\
\hline 1 & 9 & 10 & 0 & 10 & 27863.020 & 0.055 & 0.050 & 1.0 & -11.523 & $1.6 \mathrm{E}-3$ & \\
\hline 0 & 10 & 10 & 1 & 9 & 15085.470 & 0.037 & 0.050 & 0.4 & 0.165 & $4.4 E-3$ & \\
\hline 1 & 10 & 10 & 2 & 9 & 40575.680 & -0.005 & 0.050 & -0.1 & 3.827 & 4. OE-3 & \\
\hline 1 & 9 & 10 & 2 & 8 & 36461.840 & -0.149 & 0.050 & -1.7 & 10.002 & $-3.2 E-3$ & \\
\hline 1 & 10 & 11 & 0 & 11 & 32092.680 & 0.049 & 0.050 & 0.7 & -15.180 & $2.7 E-3$ & \\
\hline 0 & 11 & 11 & 1 & 10 & 15572.720 & 0.031 & 0.050 & 0.3 & -0.673 & $7.8 E-3$ & \\
\hline 1 & 10 & 11 & 2 & 9 & 36121.450 & -0.050 & 0.050 & -0.6 & 13.602 & $-5.3 E-3$ & \\
\hline 1 & 11 & 12 & 0 & 12 & 36342.140 & -0.070 & 0.050 & -1.0 & -19.497 & $4.5 E-3$ & \\
\hline 0 & 12 & 12 & 1 & 11 & 16115.420 & -0.011 & 0.050 & -0.1 & -1.999 & 0.013 & \\
\hline 1 & 11 & 12 & 2 & 10 & 35767.820 & -0.009 & 0.050 & -0.1 & 17.596 & $-8 \cdot 3 E-3$ & \\
\hline 1 & 12 & 13 & 0 & 13 & 40608.590 & 0.038 & 0.050 & 0.6 & -24.508 & 7. $0 E-3$ & \\
\hline 0 & 13 & 13 & 1 & 12 & 16716.320 & 0.026 & 0.050 & 0.3 & -3.941 & 0.021 & \\
\hline 1 & 13 & 13 & 2 & 12 & 42009.570 & 0.053 & 0.050 & 0.6 & 6.947 & 0.018 & \\
\hline 1 & 12 & 13 & 2 & 11 & 35405.500 & 0.058 & 0.050 & 0.7 & 21.963 & -0.012 & \\
\hline 1 & 13 & 14 & 0 & 14 & 44888.270 & -0.092 & 0.050 & 9.9 & -30.241 & 0.010 & \\
\hline 1 & 13 & 14 & 2 & 12 & 35039.120 & 0.046 & 0.050 & 0.5 & 26.661 & -0.018 & \\
\hline 1 & 14 & 15 & 0 & 15 & 49178.270 & 0.033 & 0.050 & 9.9 & -36.712 & 0.015 & \\
\hline 1 & 14 & 15 & 2 & 13 & 34673.760 & 0.075 & 0.050 & 0.8 & 31.630 & -0.025 & \\
\hline 1 & 16 & 16 & 2 & 15 & 43810.650 & 0.058 & 0.050 & 0.7 & 7.038 & 0.061 & \\
\hline 1 & 15 & 16 & 2 & 14 & 34314.520 & 0.107 & 0.050 & 1.2 & 36.789 & -0.033 & \\
\hline 0 & 17 & 17 & 1 & 16 & 19754.680 & -0.109 & 0.050 & -1.2 & -21.122 & 0.111 & \\
\hline 1 & 17 & 17 & 2 & 16 & 44493.010 & 0.005 & 0.050 & 0.1 & 5.966 & 0.087 & \\
\hline 0 & 18 & 18 & 1 & 17 & 20686.020 & -0.087 & 0.050 & $-1 \cdot 0$ & -28.735 & 0.159 & \\
\hline
\end{tabular}




\begin{tabular}{|c|c|c|c|c|c|c|c|c|c|c|c|}
\hline 18 & 1 & 18 & 18 & 2 & 17 & 45216.640 & 0.130 & 0.050 & 1.5 & 4.169 & 0.121 \\
\hline 19 & 0 & 19 & 19 & 1 & 18 & 21690.760 & 0.066 & 0.050 & 0.7 & -38.113 & 0.222 \\
\hline 19 & 1 & 19 & 19 & 2 & 18 & 45981.160 & 0.069 & 0.050 & 0.8 & 1.534 & 0.167 \\
\hline 20 & 0 & 20 & 20 & 1 & 19 & 22770.170 & -0.193 & 0.050 & -2.2 & -49.492 & 0.307 \\
\hline 20 & 1 & 19 & 20 & 2 & 18 & 3044.380 & -0.042 & 0.050 & -0.5 & 56.841 & -0.075 \\
\hline 1 & 0 & 21 & 21 & 1 & 20 & 23926.500 & -0.038 & 0.050 & -0.4 & -63.111 & 0.417 \\
\hline 1 & 1 & 21 & 21 & 2 & 20 & 7633.260 & 0.076 & 0.050 & 0.9 & -6.722 & 0.301 \\
\hline 1 & 1 & 20 & 21 & 2 & 19 & 2795.130 & -0.033 & 0.050 & -0.4 & 60.868 & -0.083 \\
\hline 22 & 0 & 22 & 22 & 1 & 21 & 5160.170 & -0.030 & 0.050 & -0.3 & -79.207 & 0.558 \\
\hline 22 & 1 & 21 & 22 & 2 & 20 & 32583.410 & -0.053 & 0.050 & -0.6 & 64.079 & -0.086 \\
\hline 23 & 0 & 23 & 23 & 1 & 22 & 26471.850 & 0.015 & 0.050 & 0.2 & -98.010 & 0.737 \\
\hline 23 & 1 & 22 & 23 & 2 & 21 & 32414.100 & -0.054 & 0.050 & -0.6 & 66.220 & -0.083 \\
\hline 4 & 0 & 24 & 24 & 1 & 23 & 7861.400 & 0.005 & 0.050 & 0.1 & -119.734 & 0.961 \\
\hline 24 & 1 & 24 & 24 & 2 & 23 & 50416.160 & -0.013 & 0.050 & -0.2 & -28.447 & 0.662 \\
\hline 24 & 1 & 23 & 24 & 2 & 22 & 32291.870 & 0.032 & 0.050 & 0.4 & 67.018 & -0.070 \\
\hline 25 & 0 & 25 & 25 & 1 & 24 & 9327.970 & -0.303 & 0.050 & -3.6 & -144.575 & 1.239 \\
\hline 26 & 0 & 26 & 26 & 1 & 25 & 30871.160 & -0.135 & 0.050 & -1.6 & -172.702 & 1.579 \\
\hline 26 & 1 & 25 & 26 & 2 & 24 & 32205.270 & -0.033 & 0.050 & -0.4 & 63.377 & 7. $3 E-3$ \\
\hline 27 & 0 & 27 & 27 & 1 & 26 & 32488.710 & -0.008 & 0.050 & -0.1 & -204.255 & 1.991 \\
\hline 27 & 1 & 26 & 27 & 2 & 25 & 32249.060 & 0.088 & 0.050 & 1.0 & 58.286 & 0.082 \\
\hline 28 & 0 & 28 & 28 & 1 & 27 & 34178.260 & 0.011 & 0.050 & 0.1 & -239.336 & 2.486 \\
\hline 28 & 1 & 27 & 28 & 2 & 26 & 32355.360 & -0.049 & 0.050 & -0.5 & 50.544 & 0.191 \\
\hline 29 & 0 & 29 & 29 & 1 & 28 & 35937.200 & 0.126 & 0.050 & 1.6 & -278.013 & 3.075 \\
\hline 29 & 1 & 28 & 29 & 2 & 27 & 32527.890 & -0.008 & 0.050 & -0.1 & 39.775 & 0.344 \\
\hline 30 & 0 & 30 & 30 & 1 & 29 & 37762.020 & 0.125 & 0.050 & 1.7 & -320.310 & 3.769 \\
\hline 30 & 1 & 29 & 30 & 2 & 28 & 32769.500 & 0.024 & 0.050 & 0.3 & 25.582 & 0.550 \\
\hline 31 & 1 & 30 & 31 & 2 & 29 & 33082.920 & -0.034 & 0.050 & -0.4 & 7.547 & 0.822 \\
\hline 32 & 1 & 31 & 32 & 2 & 30 & 33470.910 & -0.007 & 0.050 & -0.1 & -14.765 & 1.175 \\
\hline 33 & 1 & 32 & 33 & 2 & 31 & 33935.670 & -0.073 & 0.050 & -0.8 & -41.807 & 1.625 \\
\hline 34 & 0 & 34 & 34 & 1 & 33 & 45641.400 & 0.234 & 0.050 & 9.9 & -524.767 & 7.838 \\
\hline 34 & 1 & 33 & 34 & 2 & 32 & 4479.600 & 0.001 & 0.050 & 0.0 & -74.043 & 2.189 \\
\hline 35 & 1 & 34 & 35 & 2 & 33 & 5104.430 & -0.008 & 0.050 & -0.1 & -111.949 & 2.888 \\
\hline 36 & 1 & 35 & 36 & 2 & 34 & 5812.000 & 0.011 & 0.050 & 0.1 & -155.999 & 3.746 \\
\hline 37 & 1 & 36 & 37 & 2 & 35 & 36603.710 & -0.030 & 0.050 & -0.4 & -206.667 & 4.788 \\
\hline 38 & 1 & 37 & 38 & 2 & 36 & 7480.890 & -0.026 & 0.050 & -0.3 & -264.409 & 6.041 \\
\hline 39 & 1 & 38 & 39 & 2 & 37 & 38444.340 & -0.108 & 0.050 & -1.4 & -329.658 & 7.535 \\
\hline 40 & 1 & 39 & 40 & 2 & 38 & 39495.150 & 0.202 & 0.050 & 2.7 & -402.813 & 9.302 \\
\hline 41 & 1 & 40 & 41 & 2 & 39 & 40632.680 & 0.008 & 0.050 & 0.1 & -484.223 & 11.376 \\
\hline 42 & 1 & 41 & 42 & 2 & 40 & 1857.600 & 0.106 & 0.050 & 1.5 & -574.179 & 13.794 \\
\hline 43 & 1 & 42 & 43 & 2 & 41 & 3168.840 & -0.040 & 0.050 & -0.6 & -672.904 & 16.591 \\
\hline 44 & 2 & 42 & 44 & 3 & 41 & 0003.090 & -0.016 & 0.050 & -0.9 & 532.008 & -4.687 \\
\hline 45 & 1 & 44 & 45 & 2 & 43 & 46046.980 & -0.045 & 0.050 & -0.7 & -897.138 & 23.476 \\
\hline 46 & 2 & 44 & 46 & 3 & 43 & 49478.040 & -0.048 & 0.050 & -1.0 & 488.057 & -3.719 \\
\hline 47 & 1 & 46 & 47 & 2 & 45 & 49253.840 & -0.129 & 0.050 & 9.9 & -1156.968 & 32.338 \\
\hline 47 & 2 & 45 & 47 & 3 & 44 & 9312.620 & -0.217 & 0.050 & -3.7 & 452.308 & -2.831 \\
\hline 48 & 1 & 47 & 48 & 2 & 46 & 50974.480 & -0.182 & 0.050 & 9.9 & -1299.820 & 37.603 \\
\hline 49 & 2 & 47 & 49 & 3 & 46 & 49195.560 & -0.121 & 0.050 & -1.8 & 349.382 & 4. $3 E-3$ \\
\hline 50 & 2 & 48 & 50 & 3 & 47 & 49250.320 & -0.027 & 0.050 & -0.4 & 280.668 & 2.068 \\
\hline 52 & 2 & 50 & 52 & 3 & 49 & 49600.300 & 0.061 & 0.050 & 0.8 & 104.940 & 7.813 \\
\hline 53 & 2 & 51 & 53 & 3 & 50 & 49900.340 & 0.153 & 0.050 & 2.3 & -3.559 & 11.640 \\
\hline 54 & 2 & 52 & 54 & 3 & 51 & 50286.130 & -0.005 & 0.050 & -0.1 & -126.775 & 16.210 \\
\hline 55 & 2 & 53 & 55 & 3 & 52 & 50759.980 & 0.253 & 0.050 & 9.9 & -265.398 & 21.609 \\
\hline
\end{tabular}

rms deviation: 1.9741 time (sec): 0.11 Gramdet.: $8.5 \mathrm{E}-11$

Rotational constants (MHz) and kappa:

$\begin{array}{ccccc} & 14755.767 & 1992.473727 & 1914.325089 & -0.98782868 \\ +- & 0.022 & 0.0038 & 0.0038 & 0.00000006\end{array}$

Inertial constants and defect ( $\mathrm{A} \star * 2)$ : $\begin{array}{llll}34.249598 & 253.6440353 & 263.9985649 & -23.89507\end{array}$ 


$\begin{array}{lccccc}+- & 0.000050 & 0.00049 & 0.00052 & 0.00007 \\ \text { Quartic distortion constants: } & & & & \\ & 1.6209 & -40.5173 & 405.71 & 0.2511252 & 3.5386 \\ +- & 0.013 & 0.029 & 5.7 & 0.00011 & 0.038\end{array}$

Standard distortion constants $(\mathrm{kHz})$ :

$\begin{array}{llllll}-1467.271 & -8.492580 & -4.474577 & 89.71005 & -6.483578 & 59.39219\end{array}$

Sextic and higher distortion constants:

\begin{tabular}{|c|c|c|c|c|c|}
\hline & 0 & 0 & 0 & 0 & 0.00293864 \\
\hline+- & fixed & fixed & fixed & fixed & 0.000034 \\
\hline+- & fixed & fixed & fixed & fixed & fixed \\
\hline
\end{tabular}

Significance estimate for fixed constants:

$\begin{array}{rlllllll}9: & 9.0173 \mathrm{E}-3 & 10: & 5.6819 \mathrm{E}-3 & 11: & 2.3329 \mathrm{E}-6 & 12: & 2.2081 \mathrm{E}-2 \\ 14: & 3.6781 \mathrm{E}-2 & 15: & 5.8942 \mathrm{E}-2 & 16: & 8.3766 \mathrm{E}-2 & 17: & 5.6819 \mathrm{E}-3 \\ 18: & 2.2081 \mathrm{E}-2 & 19: & 5.8942 \mathrm{E}-2 & 20: & 5.6819 \mathrm{E}-3 & 21: & 5.8942 \mathrm{E}-2 \\ 22: & 5.8942 \mathrm{E}-2 & & & & & \end{array}$

Uncorrelation estimate for fixed constants:

$\begin{array}{rlllllll}9: & 1.0617 \mathrm{E}-1 & 10: & 8.7023 \mathrm{E}-2 & 11: & 0.0000 \mathrm{E}-9 & 12: & 1.4066 \mathrm{E}-1 \\ 14: & 1.3803 \mathrm{E}-1 & 15: & 1.5013 \mathrm{E}-1 & 16: & 1.8183 \mathrm{E}-1 & 17: & 8.7023 \mathrm{E}-2 \\ 18: & 1.4066 \mathrm{E}-1 & 19: & 1.5013 \mathrm{E}-1 & 20: & 8.7023 \mathrm{E}-2 & 21: & 1.5013 \mathrm{E}-1\end{array}$

Significant Digits and Correlation Matrix:

8

$\begin{array}{rrrrrrrr}-0.108 & -0.082 & -0.245 & -0.801 & 0.933 & -0.194 & 0.461 & 0.109 \\ 10 & 0.997 & 0.928 & 0.241 & -0.263 & 0.146 & -0.157 & 0.043 \\ & 10 & 0.921 & 0.219 & -0.238 & 0.076 & -0.150 & -0.017 \\ & 5 & 0.351 & -0.381 & 0.176 & -0.236 & 0.026 \\ & & & 6 & -0.926 & 0.312 & -0.791 & -0.111 \\ & & & & 5 & -0.236 & 0.556 & 0.100 \\ & & & & & & -0.282 & 0.852 \\ & & & & & & & 0.216\end{array}$


TABLE 5S: Allylgermane-70 Ground vib. state

Calculation by ROTFIT-TPW-1.5 of 29-MAY-1995 (with weights)

Watson A-reduction:

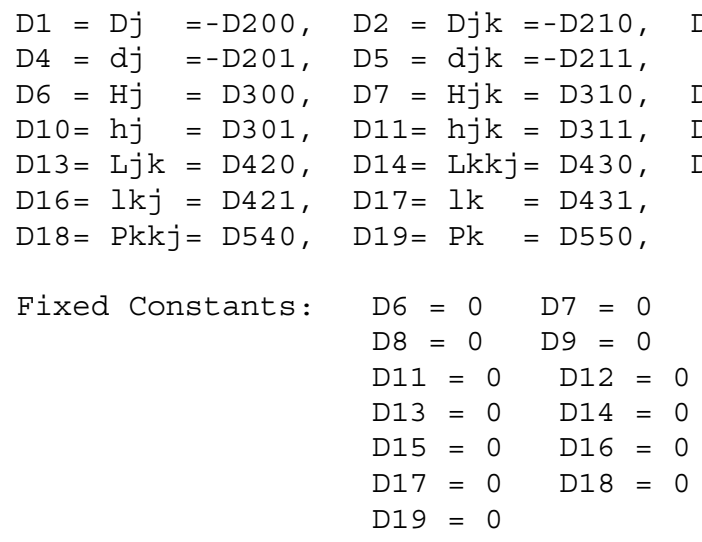

Total number: 115

\begin{tabular}{|c|c|c|c|c|c|c|c|c|c|c|c|c|}
\hline & \multicolumn{4}{|c|}{ Transition } & \multicolumn{2}{|c|}{ obs. frequency } & $\begin{array}{l}\text { obs.- } \\
\text { calc. }\end{array}$ & weight & $t$ & \multicolumn{3}{|c|}{ distortion corrections } \\
\hline 0 & 0 & 0 & 1 & 1 & 1 & 16691.994 & -0.008 & 0.0030 & 9.9 & -0.304 & $-2.3 E-8$ & \\
\hline 1 & 0 & 1 & 1 & 1 & 0 & 12846.041 & -0.001 & 0.0030 & 9.9 & -0.306 & $2.3 E-8$ & \\
\hline 2 & 0 & 2 & 2 & 1 & 1 & 12920.749 & -0.001 & 0.0030 & 9.9 & -0.155 & $6.1 \mathrm{E}-7$ & \\
\hline 3 & 0 & 3 & 3 & 1 & 2 & 13033.402 & -0.001 & 0.0030 & -0.8 & 0.057 & 4. $9 E-6$ & \\
\hline 3 & 1 & 2 & 3 & 2 & 1 & 38202.510 & -0.068 & 0.050 & -0.7 & -4.222 & $-4.8 E-6$ & \\
\hline 3 & 0 & 3 & 4 & 1 & 4 & 28120.110 & 0.363 & 0.050 & 3.2 & 0.148 & $-2.3 E-5$ & \\
\hline 4 & 0 & 4 & 4 & 1 & 3 & 13184.705 & -0.000 & 0.0030 & -0.1 & 0.311 & $2.3 E-5$ & \\
\hline 5 & 0 & 5 & 5 & 1 & 4 & 13375.595 & 0.001 & 0.0030 & 0.2 & 0.582 & $7.8 E-5$ & \\
\hline 5 & 0 & 5 & 5 & 1 & 4 & 13375.740 & 0.146 & 0.050 & 1.1 & 0.582 & $7.8 E-5$ & \\
\hline 5 & 1 & 5 & 5 & 2 & 4 & 38983.300 & -0.121 & 0.050 & $-1 \cdot 3$ & -2.310 & $7.6 E-5$ & \\
\hline 5 & 1 & 4 & 5 & 2 & 3 & 37881.640 & -0.087 & 0.050 & -0.9 & -1.794 & $-7.3 E-5$ & \\
\hline 5 & 0 & 5 & 6 & 1 & 6 & 35557.780 & -0.043 & 0.050 & -0.4 & 0.410 & $-2.1 E-4$ & \\
\hline 6 & 0 & 6 & 6 & 1 & 5 & 13607.235 & -0.000 & 0.0030 & 9.9 & 0.837 & 2. $2 \mathrm{E}-4$ & \\
\hline 6 & 0 & 6 & 6 & 1 & 5 & 13607.020 & -0.215 & 0.050 & -1.7 & 0.837 & 2. $2 E-4$ & \\
\hline 6 & 1 & 6 & 6 & 2 & 5 & 39208.410 & -0.080 & 0.050 & -0.8 & -1.050 & $2.1 E-4$ & \\
\hline 6 & 1 & 5 & 6 & 2 & 4 & 37673.170 & 0.017 & 0.050 & 0.2 & -0.103 & $-2.0 E-4$ & \\
\hline 6 & 0 & 6 & 7 & 1 & 7 & 39225.350 & -0.031 & 0.050 & -0.3 & 0.490 & $-4.9 E-4$ & \\
\hline 7 & 0 & 7 & 7 & 1 & 6 & 13881.014 & 0.000 & 0.0030 & 9.9 & 1.034 & $5.2 E-4$ & \\
\hline 7 & 0 & 7 & 7 & 1 & 6 & 13881.010 & -0.004 & 0.050 & -0.0 & 1.034 & 5. $2 \mathrm{E}-4$ & \\
\hline 7 & 1 & 6 & 7 & 2 & 5 & 37435.180 & -0.019 & 0.050 & -0.2 & 1.936 & $-4.5 E-4$ & \\
\hline 7 & 0 & 7 & 8 & 1 & 8 & 42860.450 & 0.071 & 0.050 & 0.8 & 0.528 & $-1.0 E-3$ & \\
\hline 8 & 0 & 8 & 8 & 1 & 7 & 14198.460 & -0.069 & 0.050 & -0.5 & 1.121 & 1.1E-3 & \\
\hline 8 & 0 & 8 & 9 & 1 & 9 & 46464.510 & 0.068 & 0.050 & 0.7 & 0.518 & $-2.0 E-3$ & \\
\hline 9 & 0 & 9 & 9 & 1 & 8 & 14561.420 & -0.163 & 0.050 & -1.3 & 1.034 & $2.2 E-3$ & \\
\hline 9 & 1 & 9 & 9 & 2 & 8 & 40110.930 & -0.099 & 0.050 & -1.0 & 3.289 & $2.1 \mathrm{E}-3$ & \\
\hline 9 & 1 & 8 & 9 & 2 & 7 & 36881.360 & -0.037 & 0.050 & -0.3 & 7.144 & $-1.8 E-3$ & \\
\hline 9 & 0 & 9 & 10 & 1 & 10 & 50039.640 & 0.201 & 0.050 & 2.2 & 0.456 & $-3.6 \mathrm{E}-3$ & \\
\hline 9 & 1 & 9 & 10 & 0 & 10 & 27916.530 & -0.077 & 0.050 & -0.9 & -11.555 & 1. $5 \mathrm{E}-3$ & \\
\hline 10 & 0 & 10 & 10 & 1 & 9 & 14972.190 & 0.028 & 0.050 & 0.2 & 0.699 & $4.2 E-3$ & \\
\hline 10 & 1 & 10 & 10 & 2 & 9 & 40488.020 & -0.067 & 0.050 & -0.7 & 4.765 & $3.8 E-3$ & \\
\hline 10 & 1 & 9 & 10 & 2 & 8 & 36571.570 & 0.052 & 0.050 & 0.5 & 10.346 & $-3.1 E-3$ & \\
\hline 11 & 0 & 11 & 11 & 1 & 10 & 15432.030 & -0.390 & 0.050 & -3.1 & 0.024 & $7.4 \mathrm{E}-3$ & \\
\hline 11 & 1 & 11 & 11 & 2 & 10 & 40903.480 & -0.001 & 0.050 & -0.0 & 6.160 & $6.6 E-3$ & \\
\hline 11 & 1 & 10 & 11 & 2 & 9 & 36244.090 & -0.084 & 0.050 & -0.8 & 13.958 & $-5.1 E-3$ & \\
\hline 11 & 1 & 11 & 12 & 0 & 12 & 36395.760 & -0.064 & 0.050 & -0.8 & -19.576 & $4.4 E-3$ & \\
\hline
\end{tabular}




\begin{tabular}{|c|c|c|c|c|c|c|c|c|c|c|c|}
\hline 12 & 0 & 12 & 12 & 1 & 11 & 15944.680 & 0.029 & 0.050 & 0.2 & -1.095 & 0.012 \\
\hline 12 & 1 & 11 & 12 & 2 & 10 & 35903.090 & -0.050 & 0.050 & -0.5 & 17.977 & $-8.1 E-3$ \\
\hline 12 & 1 & 12 & 13 & 0 & 13 & 40662.180 & 0.021 & 0.050 & 0.3 & -24.632 & $6.9 E-3$ \\
\hline 13 & 0 & 13 & 13 & 1 & 12 & 16511.200 & -0.060 & 0.050 & -0.5 & -2.780 & 0.020 \\
\hline 13 & 1 & 12 & 13 & 2 & 11 & 5552.520 & 0.053 & 0.050 & 0.5 & 22.384 & -0.012 \\
\hline 14 & 0 & 14 & 14 & 1 & 13 & 17134.580 & -0.143 & 0.050 & -1.2 & -5.168 & 0.032 \\
\hline 14 & 1 & 13 & 14 & 2 & 12 & 5196.610 & 0.151 & 0.050 & 1.3 & 27.149 & -0.018 \\
\hline 14 & 1 & 14 & 15 & 0 & 15 & 9233.280 & 0.028 & 0.050 & 9.9 & -36.989 & 0.015 \\
\hline 14 & 2 & 13 & 15 & 1 & 14 & 4669.770 & -0.027 & 0.050 & 9.9 & -54.581 & 0.037 \\
\hline 15 & 0 & 15 & 15 & 1 & 14 & 17817.430 & -0.115 & 0.050 & -0.9 & -8.418 & 0.048 \\
\hline 15 & 1 & 14 & 15 & 2 & 13 & 34839.800 & 0.165 & 0.050 & 1.4 & 32.220 & -0.024 \\
\hline 15 & 2 & 14 & 16 & 1 & 15 & 29142.860 & -0.036 & 0.050 & 9.9 & -66.550 & 0.053 \\
\hline 16 & 0 & 16 & 16 & 1 & 15 & 18562.130 & -0.080 & 0.050 & -0.7 & -12.702 & 0.072 \\
\hline 16 & 1 & 15 & 16 & 2 & 14 & 34486.870 & 0.180 & 0.050 & 1.6 & 37.528 & -0.033 \\
\hline 17 & 0 & 17 & 17 & 1 & 16 & 19371.100 & -0.025 & 0.050 & -0.2 & -18.211 & 0.105 \\
\hline 17 & 1 & 17 & 17 & 2 & 16 & 44207.920 & 0.101 & 0.050 & 0.9 & 8.707 & 0.083 \\
\hline 17 & 1 & 16 & 17 & 2 & 15 & 34142.590 & 0.140 & 0.050 & 1.2 & 42.980 & -0.043 \\
\hline 18 & 0 & 18 & 18 & 1 & 17 & 20246.400 & -0.163 & 0.050 & -1.4 & -25.151 & 0.150 \\
\hline 18 & 1 & 18 & 18 & 2 & 17 & 44894.700 & -0.002 & 0.050 & -0.0 & 7.339 & 0.116 \\
\hline 19 & 0 & 19 & 19 & 1 & 18 & 21190.380 & -0.219 & 0.050 & -1.9 & -33.740 & 0.211 \\
\hline 19 & 1 & 19 & 19 & 2 & 18 & 45620.620 & 0.044 & 0.050 & 0.4 & 5.189 & 0.160 \\
\hline 20 & 0 & 20 & 20 & 1 & 19 & 22205.230 & 0.183 & 0.050 & 1.6 & -44.205 & 0.290 \\
\hline 21 & 0 & 21 & 21 & 1 & 20 & 23291.470 & 0.064 & 0.050 & 0.6 & -56.780 & 0.394 \\
\hline 21 & 1 & 20 & 21 & 2 & 19 & 32950.980 & 0.092 & 0.050 & 0.8 & 63.573 & -0.088 \\
\hline 22 & 0 & 22 & 22 & 1 & 21 & 24450.800 & 0.002 & 0.050 & 0.0 & -71.700 & 0.528 \\
\hline 22 & 1 & 22 & 22 & 2 & 21 & 48031.750 & 0.248 & 0.050 & 2.2 & -7.101 & 0.379 \\
\hline 22 & 1 & 21 & 22 & 2 & 20 & 32723.740 & 0.075 & 0.050 & 0.6 & 67.542 & -0.095 \\
\hline 23 & 1 & 22 & 23 & 2 & 21 & 32534.210 & 0.149 & 0.050 & 1.2 & 70.609 & -0.096 \\
\hline 24 & 0 & 24 & 24 & 1 & 23 & 26991.020 & 0.002 & 0.050 & 0.0 & -109.480 & 0.911 \\
\hline 24 & 1 & 24 & 24 & 2 & 23 & 49831.430 & -0.323 & 0.050 & -3.0 & $-21 \cdot 380$ & 0.633 \\
\hline 24 & 1 & 23 & 24 & 2 & 22 & 32386.300 & -0.154 & 0.050 & -1.2 & 72.519 & -0.089 \\
\hline 25 & 0 & 25 & 25 & 1 & 24 & 28371.860 & 0.029 & 0.050 & 0.3 & -132.764 & 1.175 \\
\hline 25 & 1 & 25 & 25 & 2 & 24 & 50789.310 & 0.248 & 0.050 & 2.5 & -30.725 & 0.806 \\
\hline 25 & 1 & 24 & 25 & 2 & 23 & 32285.170 & 0.171 & 0.050 & 1.4 & 72.996 & -0.069 \\
\hline 26 & 0 & 26 & 26 & 1 & 25 & 29825.580 & -0.021 & 0.050 & -0.2 & -159.230 & 1.500 \\
\hline 26 & 1 & 25 & 26 & 2 & 24 & 32233.780 & 0.162 & 0.050 & 1.3 & 71.747 & -0.032 \\
\hline 27 & 0 & 27 & 27 & 1 & 26 & 31351.090 & 0.037 & 0.050 & 0.4 & -189.036 & 1.893 \\
\hline 27 & 1 & 26 & 27 & 2 & 25 & 32236.160 & 0.174 & 0.050 & 1.4 & 68.458 & 0.029 \\
\hline 28 & 0 & 28 & 28 & 1 & 27 & 32946.450 & 0.043 & 0.050 & 0.5 & -222.308 & 2.367 \\
\hline 28 & 1 & 27 & 28 & 2 & 26 & 32295.730 & 0.194 & 0.050 & 1.5 & 62.794 & 0.120 \\
\hline 29 & 0 & 29 & 29 & 1 & 28 & 34609.420 & 0.026 & 0.050 & 0.3 & -259.142 & 2.932 \\
\hline 29 & 1 & 28 & 29 & 2 & 27 & 32415.580 & 0.121 & 0.050 & 1.0 & 54.403 & 0.250 \\
\hline 30 & 0 & 30 & 30 & 1 & 29 & 36337.310 & 0.024 & 0.050 & 0.3 & -299.593 & 3.599 \\
\hline 30 & 1 & 29 & 30 & 2 & 28 & 32598.880 & 0.163 & 0.050 & 1.3 & 42.914 & 0.428 \\
\hline 31 & 0 & 31 & 31 & 1 & 30 & 38127.050 & 0.119 & 0.050 & 2.6 & -343.680 & 4.380 \\
\hline 31 & 1 & 30 & 31 & 2 & 29 & 32848.140 & 0.088 & 0.050 & 0.7 & 27.937 & 0.667 \\
\hline 32 & 0 & 32 & 32 & 1 & 31 & 39974.840 & 0.045 & 0.050 & 9.9 & -391.381 & 5.288 \\
\hline 32 & 1 & 31 & 32 & 2 & 30 & 33166.100 & 0.100 & 0.050 & 0.8 & 9.062 & 0.978 \\
\hline 33 & 1 & 32 & 33 & 2 & 31 & 33554.960 & 0.059 & 0.050 & 0.5 & -14.138 & 1.377 \\
\hline 34 & 1 & 33 & 34 & 2 & 32 & 34016.940 & 0.039 & 0.050 & 0.3 & -42.102 & 1.880 \\
\hline 34 & 1 & 33 & 34 & 2 & 32 & 34016.940 & 0.039 & 0.050 & 0.3 & -42.102 & 1.880 \\
\hline 35 & 1 & 34 & 35 & 2 & 33 & 34554.030 & 0.071 & 0.050 & 0.6 & -75.286 & 2.509 \\
\hline 36 & 1 & 35 & 36 & 2 & 34 & 35167.870 & 0.031 & 0.050 & 0.3 & -114.150 & 3.282 \\
\hline 37 & 1 & 36 & 37 & 2 & 35 & 35860.210 & 0.108 & 0.050 & 1.1 & -159.158 & 4.226 \\
\hline 38 & 1 & 37 & 38 & 2 & 36 & 36632.160 & 0.070 & 0.050 & 0.7 & -210.768 & 5.365 \\
\hline 39 & 1 & 38 & 39 & 2 & 37 & 37484.900 & -0.008 & 0.050 & -0.1 & -269.425 & 6.729 \\
\hline 40 & 1 & 39 & 40 & 2 & 38 & 38419.500 & 0.104 & 0.050 & 1.2 & -335.550 & 8.348 \\
\hline 41 & 1 & 40 & 41 & 2 & 39 & 39436.040 & -0.068 & 0.050 & -0.8 & -409.533 & 10.255 \\
\hline 42 & 1 & 41 & 42 & 2 & 40 & 40535.250 & -0.031 & 0.050 & -0.4 & -491.718 & 12.486 \\
\hline 43 & 1 & 42 & 43 & 2 & 41 & 41716.650 & -0.162 & 0.050 & -1.9 & -582.396 & 15.078 \\
\hline 43 & 2 & 41 & 43 & 3 & 40 & 50874.290 & 0.138 & 0.050 & 6.8 & 584.172 & -5.451 \\
\hline 44 & 1 & 43 & 44 & 2 & 42 & 42980.150 & -0.082 & 0.050 & -1.0 & -681.794 & 18.068 \\
\hline 44 & 2 & 42 & 44 & 3 & 41 & 50474.300 & -0.034 & 0.050 & -0.7 & 583.109 & -5.466 \\
\hline
\end{tabular}




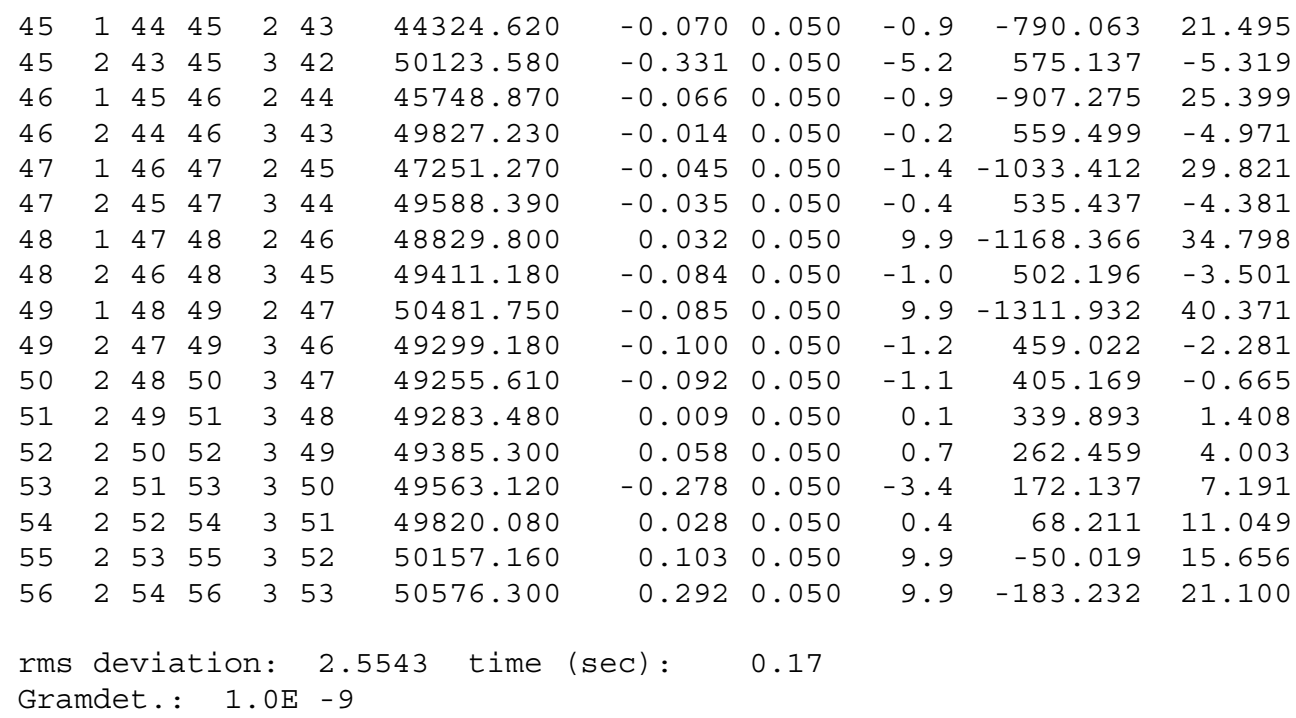

Rotational constants (MHz) and kappa:

$\begin{array}{rrcrr} & 14769.3268 & 1997.21448 & 1922.97945 & -0.98844262 \\ +- & 0.0053 & 0.0032 & 0.0031 & 0.00000005\end{array}$

Inertial constants and defect ( $\mathrm{A} * * 2)$ :

$$
\begin{array}{rcccr} 
& 34.2181525 & 253.041965 & 262.810441 & -24.44968 \\
+- & 0.000012 & 0.00040 & 0.00042 & 0.00004
\end{array}
$$

Quartic distortion constants:

$\begin{array}{cccccc} & 1.6515 & -41.3051 & 384.1 & 0.2386739 & 1.648 \\ + & 0.0093 & 0.023 & 3.1 & 0.00012 & 0.042\end{array}$

Standard distortion constants $(\mathrm{kHz})$ :

$\begin{array}{llllll}-1377.895 & -8.515547 & -4.696765 & 83.54931 & -6.606156 & 68.45871\end{array}$

Sextic and higher distortion constants:

\begin{tabular}{|c|c|c|c|c|c|}
\hline & 0 & 0 & 0 & 0 & 0.00281306 \\
\hline+- & fixed & fixed & fixed & fixed & 0.000036 \\
\hline+- & fixed & fixed & fixed & fixed & fixed \\
\hline
\end{tabular}

Significance estimate for fixed constants:

$\begin{array}{rlllllll}9: & 8.8096 \mathrm{E}-3 & 10: & 1.6463 \mathrm{E}-1 & 11: & 1.3389 \mathrm{E}-7 & 12: & 1.1486 \mathrm{E}-1 \\ 14: & 9.6315 \mathrm{E}-2 & 15: & 5.9878 \mathrm{E}-2 & 16: & 3.7737 \mathrm{E}-2 & 17: & 1.6463 \mathrm{E}-1 \\ 18: & 1.1486 \mathrm{E}-1 & 19: & 5.9878 \mathrm{E}-2 & 20: & 1.6463 \mathrm{E}-1 & 21: & 5.9878 \mathrm{E}-2 \\ 22: & 5.9878 \mathrm{E}-2 & & & & & \end{array}$

Uncorrelation estimate for fixed constants:

$\begin{array}{rlllllll}9: & 1.5885 \mathrm{E}-1 & 10: & 1.1395 \mathrm{E}-1 & 11: & 0.0000 \mathrm{E}-9 & 12: & 1.7041 \mathrm{E}-1 \\ 14: & 1.6555 \mathrm{E}-1 & 15: & 1.7723 \mathrm{E}-1 & 16: & 2.0739 \mathrm{E}-1 & 17: & 1.1395 \mathrm{E}-1 \\ 18: & 1.7041 \mathrm{E}-1 & 19: & 1.7723 \mathrm{E}-1 & 20: & 1.1395 \mathrm{E}-1 & 21: & 1.7723 \mathrm{E}-1 \\ 22: & 1.7723 \mathrm{E}-1 & & & & & & \end{array}$

Significant Digits and Correlation Matrix: 


$\begin{array}{rrrrrrrr}0.241 & 0.284 & 0.203 & -0.646 & 0.742 & -0.398 & 0.389 & -0.211 \\ 9 & 0.995 & 0.871 & 0.139 & -0.151 & 0.284 & -0.122 & 0.211 \\ & 9 & 0.864 & 0.124 & -0.127 & 0.201 & -0.124 & 0.132 \\ & & 5 & 0.116 & -0.100 & 0.282 & -0.141 & 0.199 \\ & & & 6 & -0.904 & 0.387 & -0.812 & 0.036 \\ & & & & 4 & -0.359 & 0.529 & -0.125 \\ & & & & & 7 & -0.315 & 0.881 \\ & & & & & & 4 & 0.126\end{array}$


TABLE 6S: Allylgermane-70 First ex. C2-C3 tors. state

Calculation by ROTFIT-TPW-1.5 of 29-MAY-1995 (with weights)

Watson A-reduction:

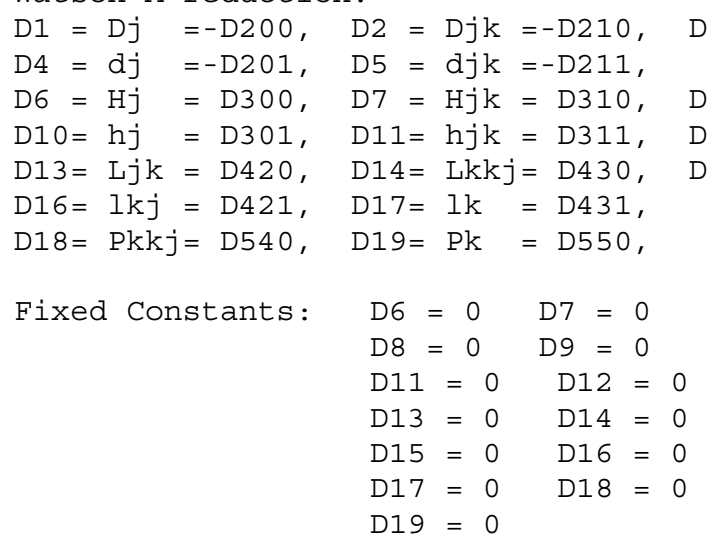

Total number: 76

Transition obs. frequency ob
$\mathrm{D} 3=\mathrm{Dk}=-\mathrm{D} 220$,

$(\mathrm{kHz})$

$\mathrm{D} 8=\mathrm{Hkj}=\mathrm{D} 320, \mathrm{D} 9=\mathrm{Hk}=\mathrm{D} 330$,

$\mathrm{D} 12=\mathrm{hk}=\mathrm{D} 321$,

$\mathrm{D} 15=\mathrm{Lk}=\mathrm{D} 440$,

$(\mathrm{Hz})$.

$\begin{array}{rrrrrrr}4 & 1 & 4 & 4 & 2 & 3 & 38783.140 \\ 5 & 1 & 4 & 5 & 2 & 3 & 37809.370 \\ 6 & 1 & 6 & 6 & 2 & 5 & 39222.720 \\ 7 & 1 & 7 & 7 & 2 & 6 & 39503.070 \\ 8 & 1 & 7 & 8 & 2 & 6 & 37054.640 \\ 8 & 0 & 8 & 9 & 1 & 9 & 46490.440 \\ 9 & 1 & 9 & 9 & 2 & 8 & 40185.110 \\ 9 & 1 & 8 & 9 & 2 & 7 & 36749.430 \\ 9 & 1 & 9 & 10 & 0 & 10 & 28180.060 \\ 10 & 0 & 10 & 10 & 1 & 9 & 15111.110 \\ 10 & 1 & 10 & 10 & 2 & 9 & 40587.280 \\ 10 & 1 & 10 & 11 & 0 & 11 & 32442.240 \\ 11 & 0 & 11 & 11 & 1 & 10 & 15605.300 \\ 11 & 1 & 10 & 11 & 2 & 9 & 36079.340 \\ 11 & 1 & 11 & 12 & 0 & 12 & 36724.510 \\ 12 & 0 & 12 & 12 & 1 & 11 & 16156.070 \\ 12 & 1 & 12 & 12 & 2 & 11 & 41514.570 \\ 12 & 1 & 11 & 12 & 2 & 10 & 35722.500 \\ 13 & 0 & 13 & 13 & 1 & 12 & 16765.770 \\ 13 & 1 & 13 & 13 & 2 & 12 & 42039.670 \\ 13 & 1 & 12 & 13 & 2 & 11 & 35357.320 \\ 13 & 1 & 13 & 14 & 0 & 14 & 45335.480 \\ 14 & 1 & 13 & 14 & 2 & 12 & 34988.570 \\ 15 & 1 & 15 & 15 & 2 & 14 & 43214.840 \\ 15 & 1 & 14 & 15 & 2 & 13 & 34621.230 \\ 16 & 0 & 16 & 16 & 1 & 15 & 18977.110 \\ 17 & 1 & 17 & 17 & 2 & 16 & 44555.620 \\ 17 & 1 & 16 & 17 & 2 & 15 & 33912.210 \\ 19 & 1 & 18 & 19 & 2 & 17 & 33273.180 \\ 20 & 0 & 20 & 20 & 1 & 19 & 22913.350 \\ 20 & 1 & 20 & 20 & 2 & 19 & 46879.290 \\ 21 & 0 & 21 & 21 & 1 & 20 & 24087.660 \\ 21 & 1 & 21 & 21 & 2 & 20 & 47736.770 \\ 21 & 1 & 20 & 21 & 2 & 19 & 32747.860 \\ 22 & 1 & 21 & 22 & 2 & 20 & 32540.800 \\ 23 & 0 & 23 & 23 & 1 & 22 & 26672.560\end{array}$

obs. - weight $t$ calc.

$$
\begin{array}{rl}
-0.062 & 0.050 \\
0.246 & 0.050 \\
-0.138 & 0.050 \\
-0.041 & 0.050 \\
-0.030 & 0.050 \\
-0.164 & 0.050 \\
-0.056 & 0.050 \\
-0.013 & 0.050 \\
0.099 & 0.050 \\
0.046 & 0.050 \\
0.006 & 0.050 \\
0.017 & 0.050 \\
-0.042 & 0.050 \\
0.095 & 0.050 \\
0.096 & 0.050 \\
0.046 & 0.050 \\
0.139 & 0.050 \\
0.019 & 0.050 \\
-0.035 & 0.050 \\
-0.107 & 0.050 \\
0.045 & 0.050 \\
-0.062 & 0.050 \\
0.094 & 0.050 \\
0.191 & 0.050 \\
0.070 & 0.050 \\
-0.181 & 0.050 \\
-0.080 & 0.050 \\
0.090 & 0.050 \\
-0.124 & 0.050 \\
-0.224 & 0.050 \\
-0.029 & 0.050 \\
-0.174 & 0.050 \\
-0.067 & 0.050 \\
-0.050 & 0.050 \\
0.001 & 0.050 \\
-0.021 & 0.050
\end{array}
$$

distortion corrections total higher fixed

$\begin{array}{rc}-3.759 & 2.5 \mathrm{E}-5 \\ -2.041 & -8.0 \mathrm{E}-5 \\ -1.498 & 2.3 \mathrm{E}-4 \\ -0.189 & 5.5 \mathrm{E}-4 \\ 4.147 & -1.0 \mathrm{E}-3 \\ 0.778 & -2.2 \mathrm{E}-3 \\ 2.563 & 2.3 \mathrm{E}-3 \\ 6.968 & -1.9 \mathrm{E}-3 \\ -11.676 & 1.7 \mathrm{E}-3 \\ 0.112 & 4.7 \mathrm{E}-3 \\ 3.900 & 4.2 \mathrm{E}-3 \\ -15.381 & 2.9 \mathrm{E}-3 \\ -0.761 & 8.3 \mathrm{E}-3 \\ 13.824 & -5.6 \mathrm{E}-3 \\ -19.752 & 4.7 \mathrm{E}-3 \\ -2.134 & 0.014 \\ 6.172 & 0.012 \\ 17.850 & -8.8 \mathrm{E}-3 \\ -4.140 & 0.023 \\ 6.959 & 0.019 \\ 22.249 & -0.013 \\ -30.626 & 0.011 \\ 26.976 & -0.019 \\ 7.415 & 0.044 \\ 31.969 & -0.026 \\ -15.576 & 0.081 \\ 5.741 & 0.092 \\ 42.389 & -0.045 \\ 52.529 & -0.067 \\ -50.949 & 0.327 \\ -2.645 & 0.240 \\ -64.914 & 0.445 \\ -7.480 & 0.320 \\ 61.002 & -0.085 \\ 64.069 & -0.088 \\ 100.662 & 0.786\end{array}$




\begin{tabular}{|c|c|c|c|c|c|c|c|c|c|c|c|}
\hline 23 & 1 & 23 & 23 & 2 & 22 & 49575.390 & 0.019 & 0.050 & 0.2 & -20.966 & 0.548 \\
\hline 23 & 1 & 22 & 23 & 2 & 21 & 32377.370 & 0.027 & 0.050 & 0.3 & 66.019 & -0.084 \\
\hline 24 & 0 & 24 & 24 & 1 & 23 & 28083.300 & -0.055 & 0.050 & -0.7 & -122.891 & 1.025 \\
\hline 24 & 1 & 24 & 24 & 2 & 23 & 50555.930 & 0.070 & 0.050 & 0.9 & -29.880 & 0.705 \\
\hline 24 & 1 & 23 & 24 & 2 & 22 & 32262.260 & 0.062 & 0.050 & 0.7 & 66.568 & -0.068 \\
\hline 25 & 0 & 25 & 25 & 1 & 24 & 29572.290 & -0.019 & 0.050 & -0.2 & -148.288 & 1. 321 \\
\hline 25 & 1 & 24 & 25 & 2 & 23 & 32199.730 & -0.023 & 0.050 & -0.3 & 65.415 & -0.035 \\
\hline 26 & 1 & 25 & 26 & 2 & 24 & 32194.140 & 0.015 & 0.050 & 0.2 & 62.234 & 0.019 \\
\hline 27 & 0 & 27 & 27 & 1 & 26 & 32779.090 & 0.088 & 0.050 & 1.1 & -209.223 & 2.123 \\
\hline 28 & 1 & 27 & 28 & 2 & 26 & 32368.430 & 0.035 & 0.050 & 0.4 & 48.390 & 0.222 \\
\hline 29 & 0 & 29 & 29 & 1 & 28 & 36275.550 & 0.074 & 0.050 & 0.9 & -284.393 & 3.276 \\
\hline 29 & 1 & 28 & 29 & 2 & 27 & 32555.120 & -0.041 & 0.050 & -0.5 & 36.975 & 0.388 \\
\hline 30 & 0 & 30 & 30 & 1 & 29 & 38124.780 & 0.097 & 0.050 & 1.3 & -327.436 & 4.013 \\
\hline 31 & 0 & 31 & 31 & 1 & 30 & 40036.280 & 0.114 & 0.050 & 1.7 & -374.100 & 4.875 \\
\hline 31 & 1 & 30 & 31 & 2 & 29 & 33143.230 & -0.011 & 0.050 & -0.1 & 3.125 & 0.910 \\
\hline 32 & 0 & 32 & 32 & 1 & 31 & 42005.730 & 0.070 & 0.050 & 1.4 & -424.315 & 5.873 \\
\hline 32 & 1 & 31 & 32 & 2 & 30 & 33549.940 & -0.032 & 0.050 & -0.4 & -20.181 & 1.293 \\
\hline 33 & 0 & 33 & 33 & 1 & 32 & 44028.600 & 0.015 & 0.050 & 9.9 & -477.973 & 7.021 \\
\hline 33 & 1 & 32 & 33 & 2 & 31 & 34035.110 & 0.033 & 0.050 & 0.4 & -48.351 & 1.780 \\
\hline 34 & 1 & 33 & 34 & 2 & 32 & 34600.630 & -0.090 & 0.050 & -1.0 & -81.860 & 2.390 \\
\hline 35 & 1 & 34 & 35 & 2 & 33 & 35248.880 & 0.034 & 0.050 & 0.4 & -121.187 & 3.146 \\
\hline 35 & 1 & 34 & 35 & 2 & 33 & 35248.880 & 0.034 & 0.050 & 0.4 & -121.187 & 3.146 \\
\hline 36 & 1 & 35 & 36 & 2 & 34 & 35981.180 & 0.013 & 0.050 & 0.2 & -166.817 & 4.073 \\
\hline 37 & 1 & 36 & 37 & 2 & 35 & 36799.170 & 0.029 & 0.050 & 0.4 & -219.223 & 5.196 \\
\hline 38 & 1 & 37 & 38 & 2 & 36 & 37704.040 & 0.091 & 0.050 & 1.2 & -278.864 & 6.546 \\
\hline 39 & 1 & 38 & 39 & 2 & 37 & 38696.550 & 0.086 & 0.050 & 1.2 & -346.171 & 8.154 \\
\hline 41 & 1 & 40 & 41 & 2 & 39 & 40946.310 & -0.079 & 0.050 & -1.2 & -505.292 & 12.283 \\
\hline 43 & 2 & 41 & 43 & 3 & 40 & 50222.110 & -0.058 & 0.050 & -1.4 & 536.120 & -4.931 \\
\hline 44 & 1 & 43 & 44 & 2 & 42 & 44979.670 & -0.133 & 0.050 & $-4 \cdot 6$ & -809.320 & 21.321 \\
\hline 44 & 2 & 42 & 44 & 3 & 41 & 49885.200 & -0.041 & 0.050 & -0.8 & 524.072 & -4.703 \\
\hline 45 & 1 & 44 & 45 & 2 & 43 & 46495.620 & -0.236 & 0.050 & 9.9 & -928.630 & 25.249 \\
\hline 45 & 2 & 43 & 45 & 3 & 42 & 49608.040 & -0.124 & 0.050 & -2.1 & 503.852 & -4.258 \\
\hline 46 & 2 & 44 & 46 & 3 & 43 & 49395.240 & -0.098 & 0.050 & -1.5 & 474.668 & -3.551 \\
\hline 49 & 2 & 47 & 49 & 3 & 46 & 49181.440 & -0.024 & 0.050 & -0.3 & 325.454 & 0.655 \\
\hline 50 & 2 & 48 & 50 & 3 & 47 & 49263.120 & 0.030 & 0.050 & 0.4 & 252.563 & 2.951 \\
\hline 51 & 2 & 49 & 51 & 3 & 48 & 49426.210 & 0.153 & 0.050 & 2.0 & 166.813 & 5.806 \\
\hline 52 & 2 & 50 & 52 & 3 & 49 & 49672.750 & -0.077 & 0.050 & -1.0 & 67.450 & 9.295 \\
\hline 53 & 2 & 51 & 53 & 3 & 50 & 50005.670 & 0.108 & 0.050 & 1.6 & -46.265 & 13.499 \\
\hline 54 & 2 & 52 & 54 & 3 & 51 & 50426.140 & 0.010 & 0.050 & 0.3 & -175.048 & 18.504 \\
\hline 55 & 2 & 53 & 55 & 3 & 52 & 50936.250 & 0.139 & 0.050 & 9.9 & -319.583 & 24.402 \\
\hline
\end{tabular}

rms deviation: 1.9685 time (sec): 0.11

Gramdet.: $3.8 \mathrm{E}-11$

Rotational constants (MHz) and kappa:

$$
\begin{array}{rrrrr}
14764.878 & 2007.62167 & 1928.46533 & -0.98766691 \\
+- & 0.025 & 0.0069 & 0.0069 & 0.00000007
\end{array}
$$

Inertial constants and defect $(u \square * * 2)$ :

$$
\begin{array}{rrrrr}
34.228462 & 251.730236 & 262.062827 & -23.89587 \\
+- & 0.000058 & 0.00087 & 0.00094 & 0.00008
\end{array}
$$

Quartic distortion constants:

$\begin{array}{cccccc} & 1.6449 & -40.7876 & 402.01 & 0.2564218 & 3.6041 \\ +- & 0.024 & 0.032 & 6.1 & 0.00012 & 0.043\end{array}$

Standard distortion constants $(\mathrm{kHz})$ :

$\begin{array}{llllll}-1451.488 & -8.630992 & -4.528244 & 90.43748 & -6.579618 & 59.55368\end{array}$

Sextic and higher distortion constants:

0 o

$0 \quad 0.00312765$ 


\begin{tabular}{|c|c|c|c|}
\hline+- & fixed & fixed & fixed \\
\hline+- & & fixed & fixed \\
\hline
\end{tabular}

Significance estimate for fixed constants:

$\begin{array}{rlllllll}9: & 5.2836 \mathrm{E}-2 & 10: & 6.8446 \mathrm{E}-3 & 11: & 1.6477 \mathrm{E}-5 & 12: & 3.0483 \mathrm{E}-2 \\ 14: & 3.8349 \mathrm{E}-2 & 15: & 6.4145 \mathrm{E}-2 & 16: & 8.5590 \mathrm{E}-2 & 17: & 6.8446 \mathrm{E}-3 \\ 18: & 3.0483 \mathrm{E}-2 & 19: & 6.4145 \mathrm{E}-2 & 20: & 6.8446 \mathrm{E}-3 & 21: & 6.4145 \mathrm{E}-2 \\ 22: & 6.4145 \mathrm{E}-2 & & & & \end{array}$

Uncorrelation estimate for fixed constants:

$\begin{array}{rlllllll}9: & 5.2760 \mathrm{E}-2 & 10: & 7.2993 \mathrm{E}-2 & 11: & 0.0000 \mathrm{E}-9 & 12: & 1.2589 \mathrm{E}-1 \\ 14: & 1.1654 \mathrm{E}-1 & 15: & 1.3413 \mathrm{E}-1 & 16: & 1.5666 \mathrm{E}-1 & 17: & 7.2993 \mathrm{E}-2 \\ 18: & 1.2589 \mathrm{E}-1 & 19: & 1.3413 \mathrm{E}-1 & 20: & 7.2993 \mathrm{E}-2 & 21: & 1.3413 \mathrm{E}-1 \\ 22: & 1.3413 \mathrm{E}-1 & & & & & & \end{array}$

Significant Digits and Correlation Matrix:

8

$\begin{array}{rrrrrrrr}0.183 & 0.202 & 0.059 & -0.821 & 0.920 & -0.238 & 0.499 & 0.088 \\ 9 & 0.999 & 0.954 & 0.090 & -0.092 & 0.073 & -0.066 & 0.025 \\ & 9 & 0.950 & 0.073 & -0.073 & 0.026 & -0.057 & -0.010 \\ & 5 & 0.194 & -0.201 & 0.111 & -0.140 & 0.015 \\ & & & 6 & -0.942 & 0.319 & -0.786 & -0.148 \\ & & & & 5 & -0.268 & 0.581 & 0.092 \\ & & & & & & -0.256 & 0.787 \\ & & & & & & & 0.349\end{array}$




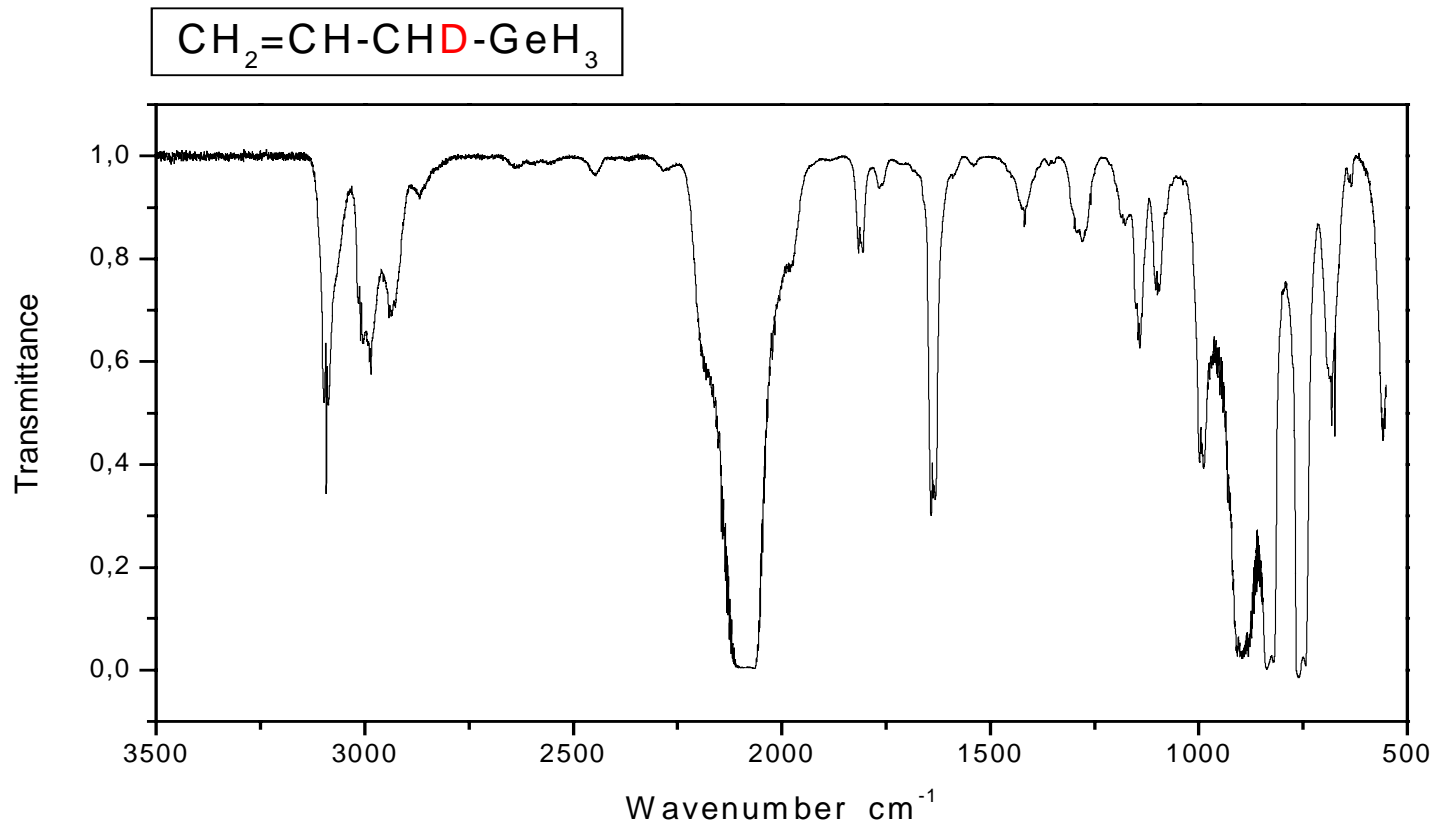

Figure 1S. Infrared spectrum of $\mathrm{CH}_{2}=\mathrm{CH}-\mathrm{CHD}-\mathrm{GeH}_{3}$ taken at a temperature of $-40{ }^{\circ} \mathrm{C}$ and a pressure of 0.404 mbar. 


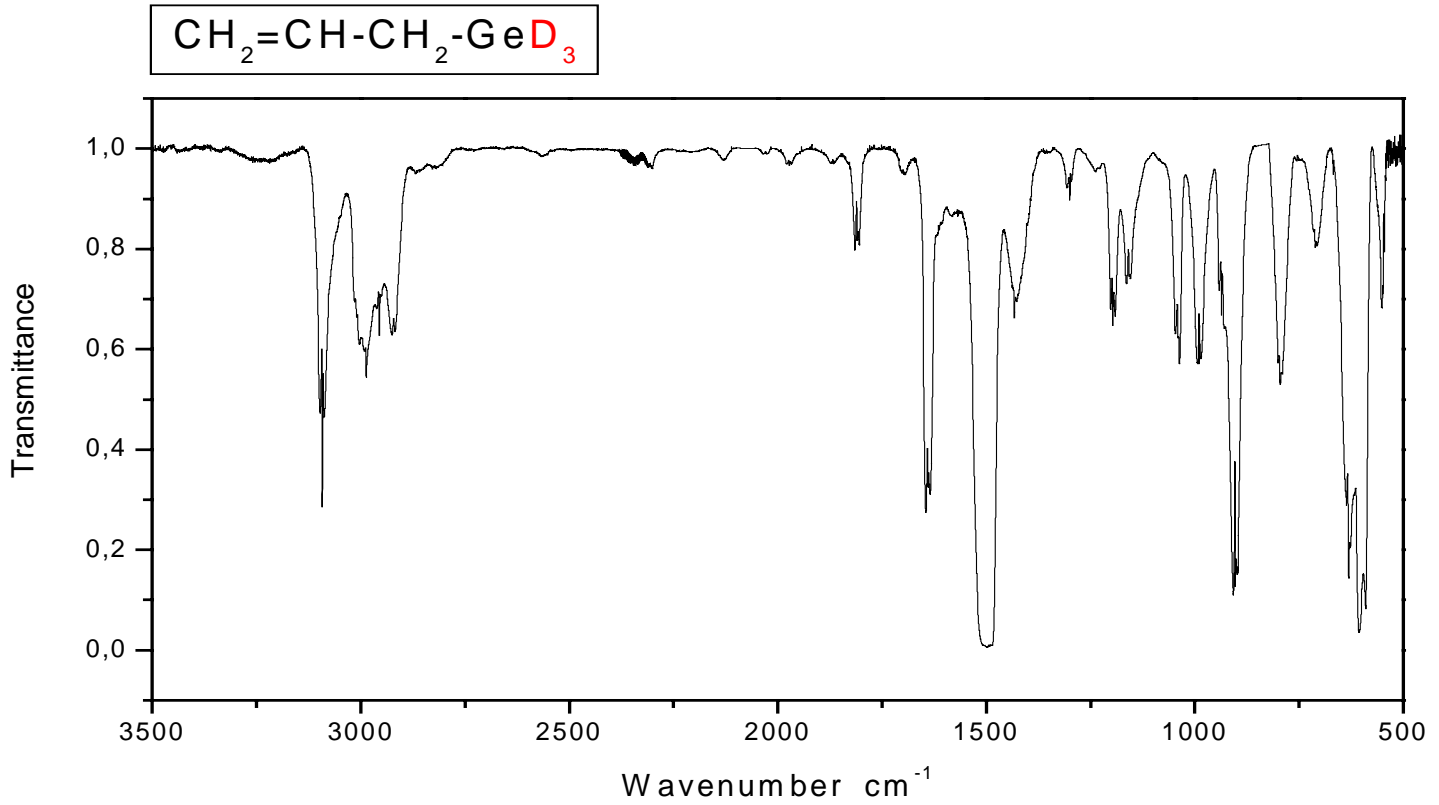

Figure 2S. Infrared spectrum of $\mathrm{CH}_{2}=\mathrm{CH}-\mathrm{CH}_{2}-\mathrm{GeD}_{3}$ taken at a temperature of $-40{ }^{\circ} \mathrm{C}$ and a pressure of 0.280 mbar. 


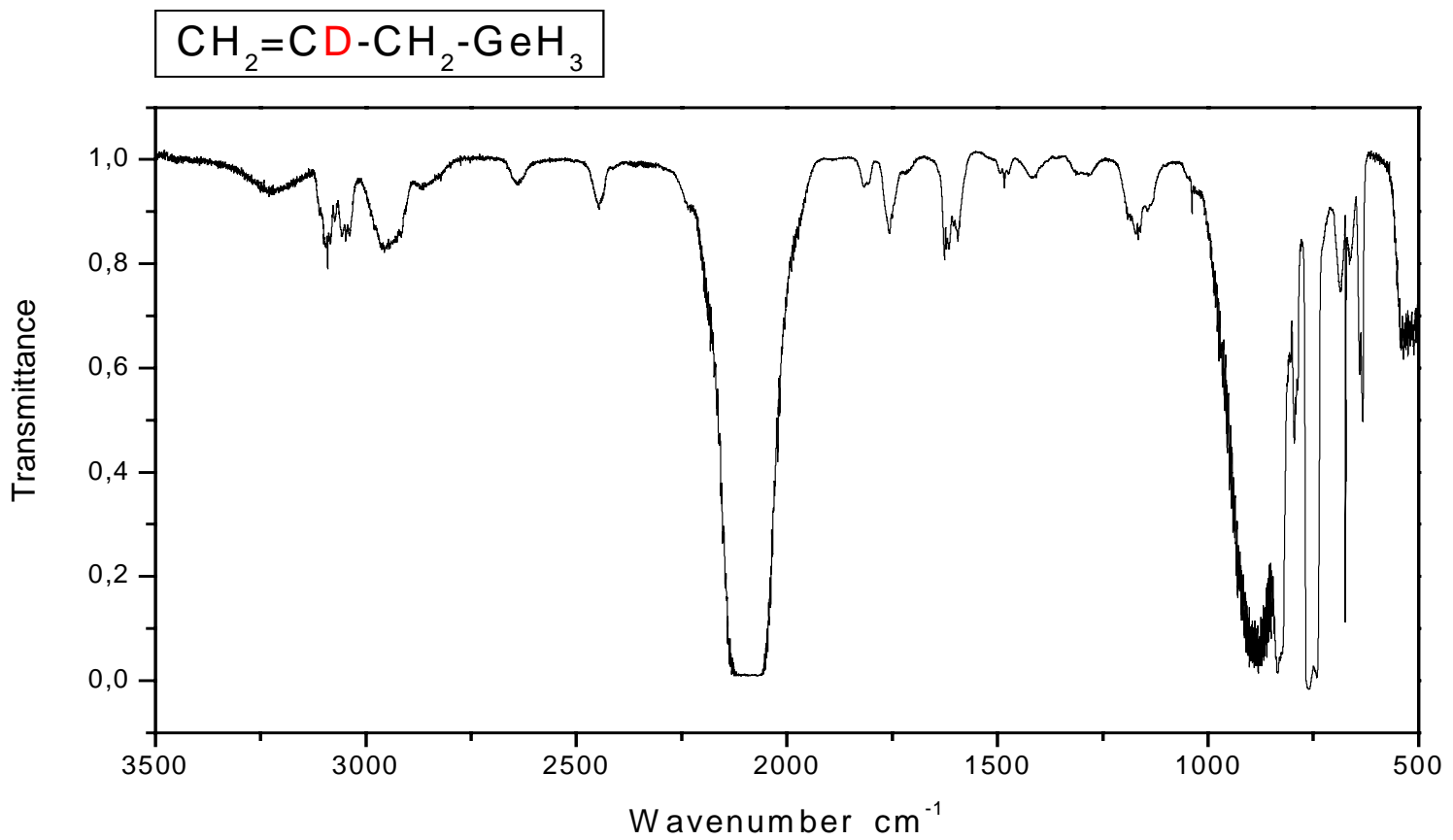

Figure 3S. Infrared spectrum of $\mathrm{CH}_{2}=\mathrm{CD}-\mathrm{CH}_{2}-\mathrm{GeH}_{3}$ taken at a temperature of $-40{ }^{\circ} \mathrm{C}$ and a pressure of 0.432 mbar. 


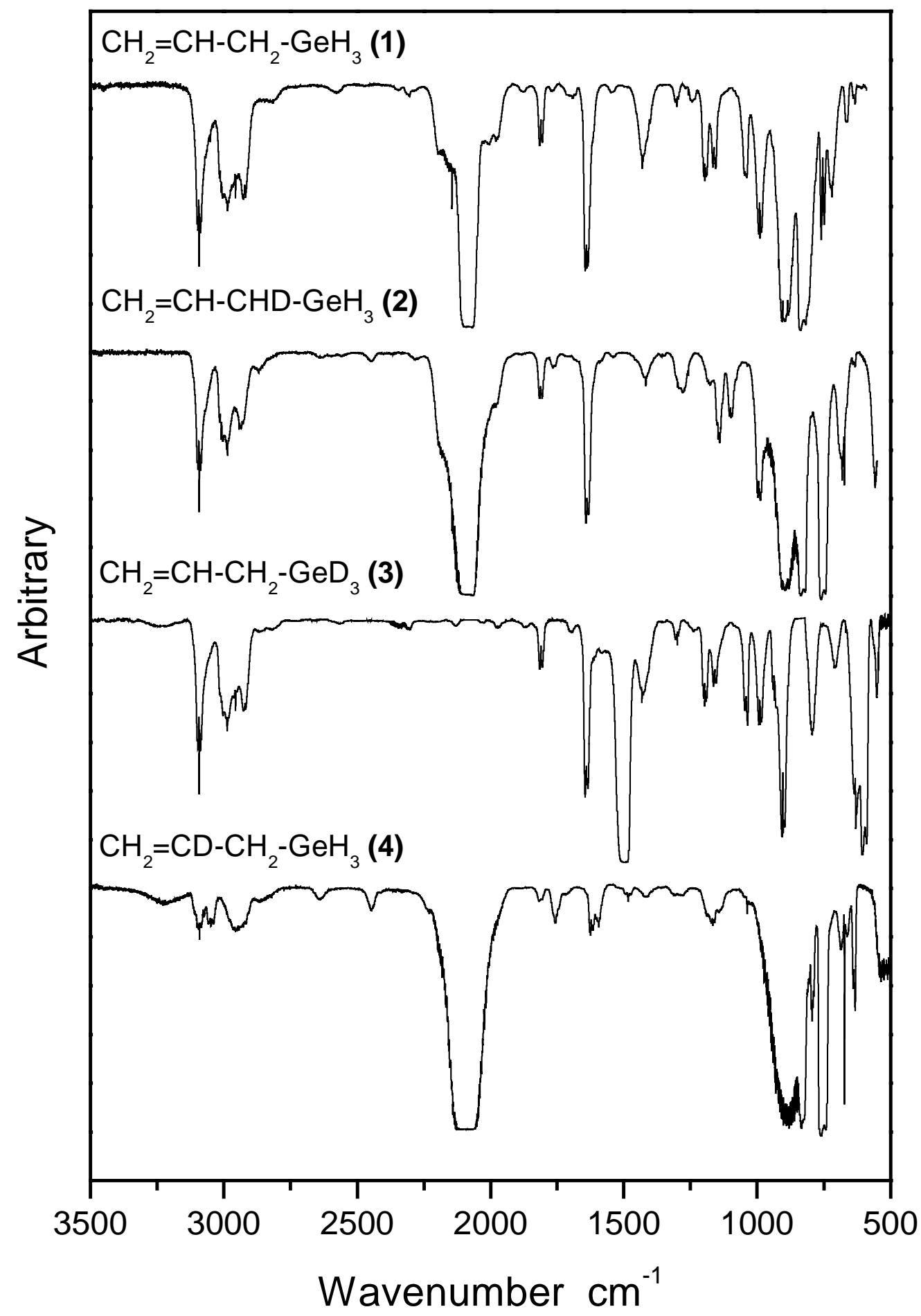

Figure 4S. Comparison of the spectra of the four species studied in this work. 
TABLE 7S: Comparison of Observed Vibrational Frequencies for Allylgermane (Gauche Form) and Its

\section{Deuterated Derivatives}

\begin{tabular}{|c|c|c|c|c|}
\hline \multirow[t]{2}{*}{ Assignments } & \multicolumn{4}{|c|}{ Frequencies (in $\mathrm{cm}^{-1}$ ) } \\
\hline & $\begin{array}{c}\mathrm{CH}_{2}=\mathrm{CH}^{-} \mathrm{CH}_{2-}- \\
\mathrm{GeH}_{3}\end{array}$ & $\begin{array}{c}\mathrm{CH}_{2}=\mathrm{CH}^{-} \mathrm{CH}_{2^{-}} \\
\mathrm{GeD}_{3}\end{array}$ & $\begin{array}{c}\mathrm{CH}_{2}=\mathrm{CH}-\mathrm{CHD}- \\
\mathrm{GeH}_{3}\end{array}$ & $\begin{array}{c}\mathrm{CH}_{2}=\mathrm{CD}-\mathrm{CH}_{2-}- \\
\mathrm{GeH}_{3}\end{array}$ \\
\hline$v_{1}=\mathrm{CH}_{2}$ antisym. stretching & 3092 & 3092 & 3092 & 3091 \\
\hline$v_{2}=\mathrm{CH}_{2}$ sym. stretching & 3052 & 3049 & 3064 & 3043 \\
\hline$v_{3}=\mathrm{CH}-$ or $=\mathrm{CD}-$ stretching & 3010 & 3010 & 3010 & ------ \\
\hline$v_{4}-\mathrm{CH}_{2^{-}}$or $-\mathrm{CHD}-$ sym. stretching & 2952 & 2952 & 2942 & 2954 \\
\hline $\begin{array}{l}v_{5}-\mathrm{CH}_{2-} \text { or }-\mathrm{CHD}-\text { antisym. } \\
\text { stretching }\end{array}$ & 2922 & 2922 & 2931 & 2982 \\
\hline$v_{6} \mathrm{GeH}_{3}$ or $-\mathrm{GeD}_{3}$ stretching & 2111 & 1505 & 2111 & 2111 \\
\hline$v_{7} \mathrm{GeH}_{3}$ or $-\mathrm{GeD}_{3}$ stretching & 2082 & 1492 & 2082 & 2082 \\
\hline$v_{8} \mathrm{GeH}_{3}$ or $-\mathrm{GeD}_{3}$ stretching & 2078 & 1492 & 2078 & 2078 \\
\hline $\begin{array}{l}2 v_{16}(=\mathrm{CH}-\text { or }=\mathrm{CD} \text { - out-of-plane } \\
\text { bending })\end{array}$ & 1973 & 1973 & 1973 & 1594 \\
\hline $2 v_{18} \quad\left(=\mathrm{CH}_{2}\right.$ wagging $)$ & 1810 & 1810 & 1810 & 1810 \\
\hline$v_{9} \quad \mathrm{C}=\mathrm{C}$ stretching & 1639 & 1640 & 1637 & 1621 \\
\hline$v_{10}=\mathrm{CH}_{2}$ deformation & 1431 & 1432 & 1417 & 1408 \\
\hline$v_{11}-\mathrm{CH}_{2}-$ or $-\mathrm{CHD}$ - deformation & 1405 & 1402 & 1354 & 1369 \\
\hline$v_{12}=\mathrm{CH}-$ or $=\mathrm{CD}-$ in-plane bending & 1299 & 1300 & 1298 & 1038 \\
\hline$v_{13}-\mathrm{CH}_{2^{-}}$or $-\mathrm{CHD}-$ wagging & 1199 & 1196 & 1146 & 1166 \\
\hline$v_{14}-\mathrm{CH}_{2}-$ or $-\mathrm{CHD}$ - twisting & 1159 & 1157 & 1099 & 1139 \\
\hline$v_{15} \quad \mathrm{C}-\mathrm{C}$ stretch & 1040 & 1040 & ----- & 1038 \\
\hline $\begin{array}{l}v_{16}=\mathrm{CH}-\text { or }-\mathrm{CHD} \text { - out-of-plane } \\
\text { bending }\end{array}$ & 989 & 990 & 994 & 791 \\
\hline$v_{17}=\mathrm{CH}_{2}$ rocking & 920 & 936 & 919 & ----- \\
\hline$v_{18}=\mathrm{CH}_{2}$ wagging & 903 & 903 & 903 & 904 \\
\hline$v_{19} \mathrm{GeH}_{3}$ or $-\mathrm{GeD}_{3}$ deformation & 883 & 631 & 884 & 884 \\
\hline$v_{20} \mathrm{GeH}_{3}$ or $-\mathrm{GeD}_{3}$ deformation & 829 & 599 & 829 & 829 \\
\hline$v_{21} \mathrm{GeH}_{3}$ or $-\mathrm{GeD}_{3}$ deformation & 754 & 548 & 751 & 751 \\
\hline$v_{22}-\mathrm{CH}_{2}-$ or $-\mathrm{CHD}$ - rocking & 720 & 711 & 685 & 682 \\
\hline$v_{23}=\mathrm{CH}_{2}$ twisting & 664 & 667 & 664 & 644 \\
\hline
\end{tabular}


Symbol used: $=\mathrm{CH}_{2}$ : vinyl group; $-\mathrm{CH}_{2}-$ : methylene group; [------] not identified 